\title{
RLNC-Aided Cooperative Compressed Sensing for Energy Efficient Vital Signal Telemonitoring
}

\author{
Aris S. Lalos, Angelos Antonopoulos, Elli Kartsakli, Member, IEEE, \\ Marco Di Renzo, Senior Member, IEEE, Stefano Tennina, Member, IEEE, \\ Luis Alonso, and Christos Verikoukis, Senior Member, IEEE
}

\begin{abstract}
Wireless Body Area Networks (WBANs) are composed of sensors that either monitor and transmit vital signals or act as relays that forward the received data to a Body Node Coordinator (BNC). In this paper, we introduce an energy efficient vital signal telemonitoring scheme, which exploits Compressed Sensing (CS) for low-complexity signal compression/reconstruction and distributed cooperation for reliable data transmission to the BNC. More specifically, we introduce a Cooperative Compressed Sensing (CCS) approach, which increases the energy efficiency of WBANs by exploiting the benefits of Random Linear Network Coding (RLNC). We study the energy efficiency gains of RLNC compared to the Store-and-Forward (FW) protocol, by providing closed-form expressions that show that the gain introduced by RLNC increases as the link failure rate increases, especially in practical scenarios with a limited number of relays. Furthermore, we propose a reconstruction algorithm that further enhances the benefits of RLNC by exploiting key characteristics of vital signals. With the aid of electrocardiographic (ECG) and electroencephalographic (EEG) data available in medical databases, extensive simulation results are illustrated, which validate our theoretical findings and show that the proposed recovery algorithm increases
\end{abstract}

Part of this work was presented at the IEEE Global Communications Conference, Austin, TX, USA, Dec. 2014.

A. Lalos, E. Kartsakli and L. Alonso are with the Department of Signal Theory and Communications (TSC) of the Technical University of Catalonia (UPC), Barcelona, Spain. E-mail: \{aristeidis.lalos, ellik, luisg\}@tsc.upc.edu

A. Antonopoulos and C. Verikoukis are with the Telecommunications Technological Centre of Catalonia (CTTC), Castelldefels, Spain. E-mail:\{aantonopoulos, cveri\}@cttc.es

M. Di Renzo is with the Laboratory of Signals and Systems (L2S), CNRS - SUPELEC - University Paris-Sud XI, Paris, France. E-mail: marco.direnzo@lss.supelec.fr

S. Tennina is with WEST Aquila srl, L'Aquila, Italy. E-mail: tennina@westaquila.it 
the energy efficiency of the body sensor nodes by $40 \%$ compared to conventional CS-based reconstruction methods.

\section{INTRODUCTION}

Wireless Body Area Networks (WBANs) consist of low-power, lightweight wireless sensors, either wearable or implantable, which are specialized to perform specific tasks. Although the primary function of these sensors is to continuously monitor human physiological signals and activities [1], they can also be employed as relays [2] that forward the data of other sensors to a Body Node Coordinator (BNC), which is also attached to the human body [2]. Wireless technologies are used to this end, providing higher flexibility, portability and offering significant advantages compared to traditional wired solutions. However, the adoption of wireless technologies raises important issues that are mainly related to the WBAN energy efficiency and reliability [1].

It has been shown that the RF power amplifier of a WBAN transmitter consumes approximately $73 \%$ of the total power [3]. Moreover, the transmission of radio signals in a WBAN, takes place near the human body that is a very lossy medium. Consequently, the link quality is very likely to be deteriorated due to the high path loss [2], which causes severe attenuation in the wireless signals, especially during different body actions (e.g., sitting, walking, running) [4]. In addition, pervasive use of WBAN increases the need for coexistence between multiple WBANs, e.g., closely-located patients in a medical ward [4, Fig. 10], causing interference that is also a threat for the reliable operation of those networks [5]. Those challenges, introduce two major requirements: i) the need for lower energy consumption at the RF part of the body nodes, and ii) the need for enhanced robustness against link failures. Apparently, the aforementioned challenges stress the need for novel signal processing algorithms and communication protocols for an energy efficient and reliable compression, transmission and reconstruction of the medical information.

The compression/reconstruction efficiency of signal processing algorithms running on sensor, relay body nodes and the BNC can be optimized by employing encoding/decoding schemes with high Compression Ratio (CR) capabilities and reduced computational requirements. However, the vast majority of biosignal (e.g., ECG and EEG) compression schemes available in the literature [6], [7], [8] charge the transmitter with most of the processing, 
thus not coping effectively with these requirements. To overcome this limitation, Compressed Sensing (CS) has recently been proposed as a viable low complexity signal processing solution for signal compression/reconstruction, providing a systematic approach for reconstructing sparse signals from a small number of random linear observations.

Besides the energy efficiency gains that can be achieved by the application of CS, cooperative transmission can provide robustness to link failures in WBANs, by allowing intermediate body nodes to act as relays [2], [9]. In practical scenarios, however, the number of potential relays is limited and the reception of data by intermediate nodes may have a non-trivial energy expenditure [2]. These remarks, motivate us to analyze the potential benefits and limitations of using cooperative relaying in WBANs, as well as to design cooperative transmission protocols that rely on a limited number of relays, while improving network energy efficiency and robustness to link failures.

\section{A. Related Work}

Several works in the literature [10], [11], [12] have exploited CS for compressing large amounts of data (e.g., temperature, light, humidity readings) originating from large-scale sensor networks and for reconstructing the original data from a small number of observations made available to a central coordination unit. Due to its appealing properties, CS has been recently applied to WBAN-oriented telemonitoring applications. In [3], [13], the authors show that the compression of ECG and EMG signals can be efficiently implemented at the biosensors by applying random linear coding to the recorded measurements, while state-of-the-art CS algorithms (e.g., LASSO) can be used at the receiver for the efficient reconstruction of the received signals. In [14], a scheme that improves the capabilities of conventional CS algorithms by exploiting key characteristics (i.e., the block sparse structure) of the transmitted signals is introduced. More specifically, the authors propose a Block Sparse Bayesian Learning (BSBL) framework for compressing/reconstructing non-sparse raw fetal ECG recordings, which improves the energy efficiency of state-of-the-art CS schemes.

Despite the insights onto the design of efficient CS-based signal encoding/ decoding algorithms for telemonitoring applications, the aforementioned works assume only direct communication between the sensor nodes and the BNC, completely ignoring the fact that body nodes are often able to communicate and cooperate with each other. In such scenarios, 
the incorporation of Random Linear Network Coding (RLNC) [15] would potentially increase the energy efficiency of telemonitoring schemes in WBANs, facilitating at the same time the application of conventional CS-based recovery algorithms, which require random linear coded data. In this context, some papers have recently applied intra-session RLNC [16] and inter-session RLNC [17], [18] concepts for encoding packets originating either from the same or from different wireless flows, respectively. Nonetheless, although these works combine RLNC-based cooperation and CS, they do not quantify the gains of RLNC compared to the conventional Store-and-Forward (FW) algorithm. More specifically, the number of relays in the network and the quality of the wireless links have an important impact on network performance and energy efficiency, which still remains unexplored. In addition, another important limitation of state-of-the-art papers is that the data transmitted in the network is usually assumed to change smoothly in time (e.g., light and temperature). However, this assumption does not hold, in general, for vital signals (e.g., ECG, EEG and EMG), whose peculiar characteristics require the development of novel CS-based recovery schemes for increasing the energy efficiency and reducing the computational complexity.

\section{B. Contribution}

In this paper, motivated by the aforementioned open issues, we introduce an intra-session RLNC-aided Cooperative Compressed Sensing (CCS) scheme for energy efficient vital signal telemonitoring and we evaluate the energy efficiency gains that can be achieved by coupling CS and RLNC in WBAN applications. In addition, we propose a novel CS-based reconstruction algorithm, which enhances the benefits of the proposed CCS scheme by taking into account specific characteristics (e.g., high temporal correlation, block sparsity) of biosignals (e.g., ECG, EEG, EMG). The contribution of this paper can be summarized as follows:

- We propose a novel biosignal telemonitoring architecture for WBANs, which exploits relaying capabilities of body sensor nodes. Both FW-based and RLNC-based cooperative communication protocols are analyzed and compared.

- We provide closed form expressions that quantify the energy efficiency gain of RLNCbased cooperation compared to FW-based cooperation in single- and multi-relay scenarios. Two main conclusions emerge from this analysis: 
1) In error-prone channels, the performance of RLNC-based schemes is better than FWbased schemes. In particular, the energy efficiency gain of RLNC increases as the packet error probability increases.

2) Increasing the number of relays in the network increases the robustness to link failures but reduces the energy efficiency gains introduced by RLNC. Thus, in order to improve the energy efficiency of RLNC-aided WBANs and to increase the robustness to link failures, a limited number of relays need to be employed.

- We present a novel low complexity reconstruction algorithm, namely Decorrelated Iterative Reweighed Group LASSO (DIG LASSO), exploits inherent block sparsity properties of typical biosignals either in the time domain or in an appropriate transform domain. Extensive simulation experiments with real medical data, demonstrate that DIG LASSO offers a higher network energy efficiency and an enhanced robustness to wireless link failures compared to state-of-the-art CS-based reconstruction algorithms (e.g., LASSO, BSBL) [3], [13], [14].

\section{Organization}

The remainder of this paper is organized as follows. In Section II, we briefly review concepts and terminology related to CS theory. In Section III, we present the system model. In Section IV, we introduce RLNC-based and FW-based cooperative relaying and we provide analytical expressions for the energy efficiency performance of each protocol. In Section V, we present the DIG LASSO reconstruction algorithm. In Section VI, the performance of the proposed scheme and protocols is evaluated and compared to state-of-the-art algorithms, by taking into account real medical data. Finally, Section VII concludes this paper.

\section{Notation}

Lower- and upper-case boldface letters are used to denote column vectors and matrices, respectively; calligraphic letters are used to denote sets; $(\cdot)^{T}$ denotes transposition; $\bmod (M, K)$ denotes the remainder of the Euclidean division of $M$ by $K ;\lfloor M / K\rfloor$ denotes the nearest integer of $M / K$ towards minus infinity; $\mathbb{E}[\cdot]$ denotes the expectation operator; $\|\mathbf{x}\|_{p}:=\left(\sum_{i=1}^{n}\left|x_{i}\right|^{p}\right)^{(1 / p)}$ for $p \geq 1$ stands for the $\ell_{p}$-norm in $\mathbb{R}^{n} ;\|\mathbf{x}\|_{0}$ denotes the $\ell_{0}$-pseudonorm that equals to the number of non-zero entries of $\mathbf{x}$. 


\section{Preliminaries on Compressed Sensing}

CS provides a systematic approach for reconstructing a sparse signal $\mathbf{x} \in \mathbb{R}^{N}$ by using only a small number of linearly combined measurements [19], [20]. A signal $\mathbf{x}$ is said to be $K_{s}$-sparse if only $K_{s}$ entries of $N$ are non-zero. The encoded measurements, $\mathbf{y} \in \mathbb{R}^{M}$, are generated using a random matrix $\mathbf{A} \in \mathbb{R}^{M \times N}$ with independent and identically distributed (i.i.d.) elements. In mathematical terms, $\mathbf{y}=\mathbf{A x}+\mathbf{z}$, where $\mathbf{z}$ is the noise vector.

\section{A. Reconstruction by Exploiting Sample Sparsity}

In the noise-free case (i.e., $\mathbf{z}=\mathbf{0}_{N}$ ), the vector $\mathbf{x}$ may be ideally recovered from the vector $\mathbf{y}$ by solving the minimization problem $\min _{x}\left\{\|\mathbf{x}\|_{0}: \mathbf{y}=\mathbf{A x}\right\}$. In order to make the signal reconstruction robust to the presence of noise, the constraint of the problem may be relaxed as $\min _{x}\left\{\|\mathbf{x}\|_{0}:\|\mathbf{y}-\mathbf{A x}\|_{2}^{2} \leq \epsilon\right\}$, where $\epsilon$ is a predefined error tolerance. This approach, however, cannot be used for practical applications, since it is computationally intractable. CS provides a solution to this issue, by replacing the $\ell_{0}$ quasi-norm with the convex $\ell_{1}$-norm and by solving a new optimization problem that can be formulated as $\min _{x}\left\{\|\mathbf{x}\|_{1}:\|\mathbf{y}-\mathbf{A x}\|_{2}^{2} \leq \epsilon\right\}$, where the $\ell_{1}$-norm is defined as $\|\mathbf{x}\|_{1}=\sum_{i=1}^{N}\left|x_{i}\right|$. By employing Lagrange relaxation, it is possible to efficiently find an solution by solving the $\ell_{1}$-regularized least square problem as follows:

$$
\hat{\mathbf{x}}:=\underset{\mathbf{x}}{\arg \min }\|\mathbf{y}-\mathbf{A x}\|_{2}^{2}+\lambda\|\mathbf{x}\|_{1},
$$

where $\lambda$ is a penalty parameter that can be tuned, to trade off the value of the ordinary least square error $\|\mathbf{y}-\mathbf{A x}\|_{2}^{2}$ and the degree of sparsity, i.e., the number of the nonzero entries, in $\mathrm{x}$.

Algorithmically, the convex optimization problem in eq. (1), known as LASSO problem, can be tackled by any generic Second-Order Cone Program (SOCP) solver. In order to achieve a good estimation of $\mathbf{x}$ (e.g., accurately estimate the $K$ largest $x_{i}$ ), the random matrix A needs to satisfy the Restricted Isometry Property (RIP) [21].

Definition 1: A matrix A satisfies the $K_{s}$-RIP with restricted isometry constant $\delta_{K}$ if, for all $M \times K$ submatrices $A_{K}$ of $A$ and for every vector $\mathbf{y}$, the following inequalities hold:

$$
\left(1-\delta_{K}\right)\|\mathbf{y}\|_{2}^{2} \leq\|\mathbf{A y}\|_{2}^{2} \leq\left(1+\delta_{K}\right)\|\mathbf{y}\|_{2}^{2} .
$$


Random matrices whose entries are i.i.d. Gaussian, Rademacher $(+1$ or -1 with probability 0.5), or, more generally, subgaussian Random Variables (RVs) satisfy this property with high probability, provided that $M=O\left(K_{s} \log \left(N / K_{s}\right)\right)[22]$.

Definition 2: An RV $X$ is said to be $c$-subgaussian if there exists a $c>0$ such that:

$$
\mathbb{E}\left[e^{X t}\right] \leq e^{c^{2} t^{2} / 2} \quad \forall t \in \mathbb{R}
$$

A $c$-subgaussian RV $X$ is denoted by $X \sim S u b\left(c^{2}\right)$.

\section{B. Reconstruction by Exploiting Block Sparsity}

A block sparse signal is a signal that consists of clusters of non-zero coefficients. A $K_{b^{-}}$ block sparse signal consists of $K_{b}$ zero blocks and, as a result, has sparsity $K_{s}=K_{b} d$, where $d$ denotes the block length. In mathematical terms, a vector $\mathbf{x}$ can be formulated as a concatenation of $R$ blocks of length $d$, as follows:

$$
\mathbf{x}=[\underbrace{x_{1}, \ldots, x_{d}}_{\mathbf{x}^{T}[1]}, \underbrace{x_{d+1}, \ldots, x_{2 d}}_{\mathbf{x}^{T}[2]}, \ldots, \underbrace{x_{N-d+1}, \ldots, x_{N}}_{\mathbf{x}^{T}[R]}]^{T},
$$

where $\mathbf{x}[i]$ denotes the $i^{\text {th }}$ block and $N=R d$.

Similar to eq. (4), the measurement matrix $\mathbf{A}$ can be represented as a concatenation of

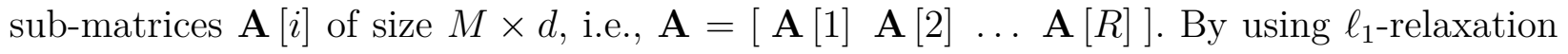
for reconstructing $\mathbf{x}$, we ignore the fact that the signal is block-sparse, i.e., the non-zero entries occur in consecutive positions. To exploit block sparsity, it is possible to reconstruct the vector $\mathbf{x}$ by solving the optimization problem as follows:

$$
\hat{\mathbf{x}}:=\underset{\mathbf{x}}{\arg \min }\left\|\mathbf{y}-\sum_{i=1}^{R} \mathbf{A}[i] \mathbf{x}[i]\right\|_{2}^{2}+\lambda \sum_{i=1}^{R}\|\mathbf{x}[i]\|_{2},
$$

which is known as group LASSO problem [23]. The authors of [24] show that the number of measurements required for robust recovery using eq. (5) is of the order of $M=$ $O\left(d K_{b}+K_{b} \log \left(R / K_{b}\right)\right)$, which is remarkably lower than $M=O\left(d K_{b} \log \left(N / K_{s}\right)\right)$ that is required by conventional CS recovery methods, e.g., by using eq. (1).

\section{Reconstruction by Exploiting Sparsity in a Transform Domain}

In many applications, a signal $\mathbf{x}$ may be sparse in a transform domain (e.g. wavelet domain) instead of the time domain. In this case, $\mathbf{x}$ can be expressed as $\mathbf{x}=\Psi \mathbf{s}$, where 


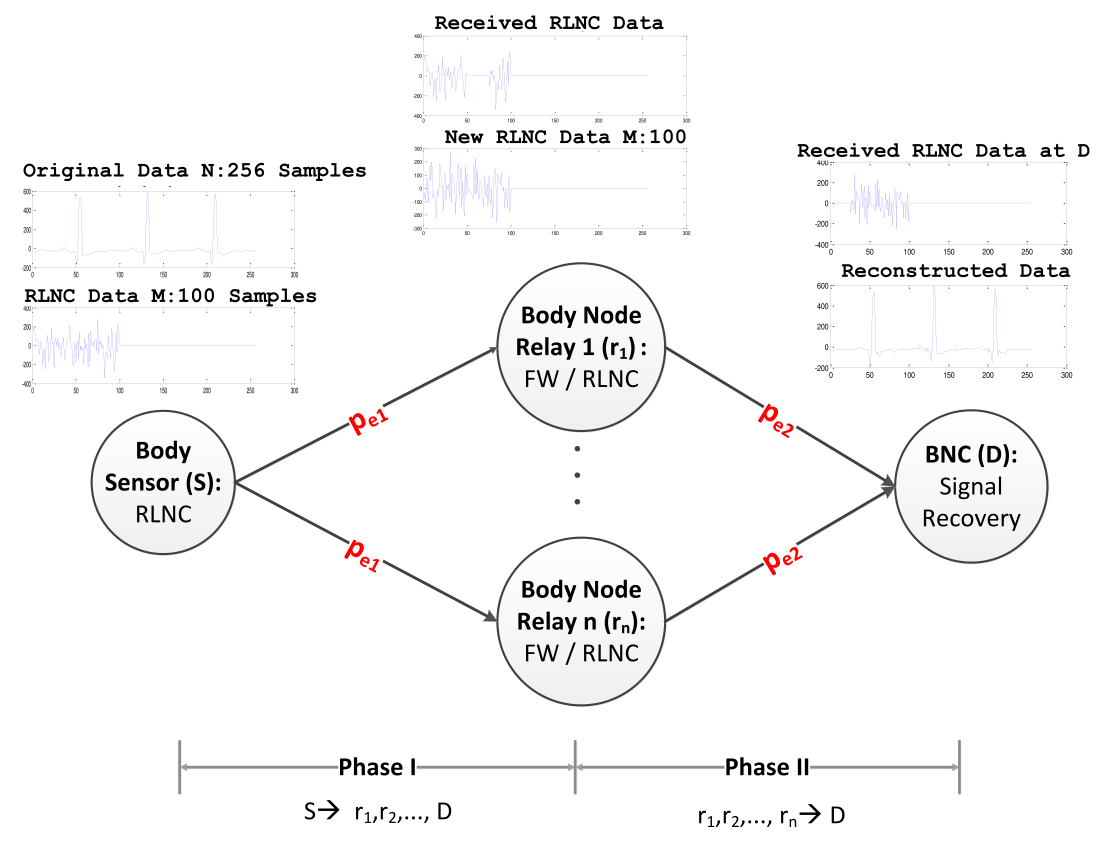

Fig. 1. Wireless Telemonitoring System Model. Source and relay nodes are wearable or implant nodes forming a $W B A N$.

$\Psi \in \mathbb{R}^{N \times N}$ is an orthonormal basis matrix of an appropriate transform domain, and $\mathbf{s}$ is a sparse representation vector. Accordingly, instead of solving eq. (1) or eq. (5), the block sparsity characteristic of $\mathbf{s}$ can be exploited by solving the following optimization problems:

$$
\begin{aligned}
& \hat{\mathbf{s}}:=\underset{\mathbf{s}}{\arg \min }\|\mathbf{y}-\mathbf{A} \Psi \mathbf{s}\|_{2}^{2}+\lambda\|\mathbf{s}\|_{1} \\
& \hat{\mathbf{s}}:=\underset{\mathbf{s}}{\arg \min }\|\mathbf{y}-\mathbf{A} \Psi \mathbf{s}\|_{2}^{2}+\lambda \sum_{i=1}^{R}\|\mathbf{s}[i]\|_{2}
\end{aligned}
$$

and the original signal in the native domain can be reconstructed by computing $\hat{\mathbf{x}}=\Psi \hat{\mathbf{s}}$.

\section{Wireless Telemonitoring System Model}

Figure 1 illustrates the telemonitoring system model under study. In particular, we consider a WBAN formed by a biosensor (source node) that records a real time vital signal (e.g., ECG or EEG) and transmits it to the BNC with the aid of a cluster of $n$ body nodes that act as relays, denoted by $r_{i}, i=1, \ldots, n$. We assume that all sensors are attached to the same body. The packet error probability of the links from the source to the relays and from the relays to the $\mathrm{BNC}$ are denoted by $p_{e 1}$ and $p_{e 2}$, respectively. In this section, we 
describe the operations performed at the sensor node as well as the considered cooperative communication protocol.

\section{A. Operations Executed at the Source}

The recorded measurements are divided in segments of $N$ samples. Each segment is represented as a vector $\mathbf{x}=\left[x_{1}, \ldots, x_{N}\right]^{T}$, where $x_{i} \in \mathbb{R}$. These measurements of bioelectric events are exposed to different sources of noise, including patients (in the form of biopotentials), electrode leads, bioamplifiers and signal transducers, etc. [25]. In this work, we assume that the recorded signal can be formulated as $\mathbf{u}=\mathbf{x}+\mathbf{z}_{s}$, where $\mathbf{u}=\left[u_{1}, \ldots, u_{N}\right]^{T}$ are noisy signal samples and $\mathbf{z}_{s}=\left[z_{1}, \ldots, z_{N}\right]^{T}$ corresponds to the noise due to the bioamplifier, which is modeled as zero mean Gaussian RV.

As mentioned in Section I, intra-session RLNC [26], [27] can serve as an encoding mechanism at the application layer [17], which produces random linear combinations of the observed data that can be exploited by CS algorithms for an efficient reconstruction of the original information. For each segment of the original signal, the source generates $M$ random linear combinations by employing a random matrix $\mathbf{A}_{s}$ of dimension $M \times N$, as follows:

$$
\mathbf{y}=\mathbf{A}_{s} \mathbf{u}=\mathbf{A}_{s} \mathbf{x}+\mathbf{z}
$$

where $\mathbf{y}=\left[y_{1}, \ldots, y_{M}\right]^{T}$ and $\mathbf{z}=\mathbf{A}_{s} \mathbf{z}_{s}$. To reduce the computational complexity of the encoding process, the coefficients $\mathbf{A}_{s i, j}$ are chosen as Rademacher RVs, i.e., $\mathbf{A}_{s i, j}= \pm 1 / \sqrt{N}$ with probability $0.5[17]$.

\section{B. Frame Structure and Communication protocol}

As far as the communication protocol is concerned (Fig. 2), we consider a time-slotted system, where the transmission of each packet $\mathbf{y}_{i}, \forall i \in[1, M]$, is performed in a communication round $(\mathrm{CR})$ that is divided into two phases. In Phase I $\left(P_{I}\right)$, the source node transmits the $M$ generated packets in $M$ distinct time slots. For each packet sent by the source to the relays, two events can occur: 1) if the received packet meets the Quality of Service (QoS) requirement set by the application layer (i.e., a target packet error probability), then it is considered as a correct packet that can be further processed by higher layers, and 2) 


\section{Communication Protocol:}

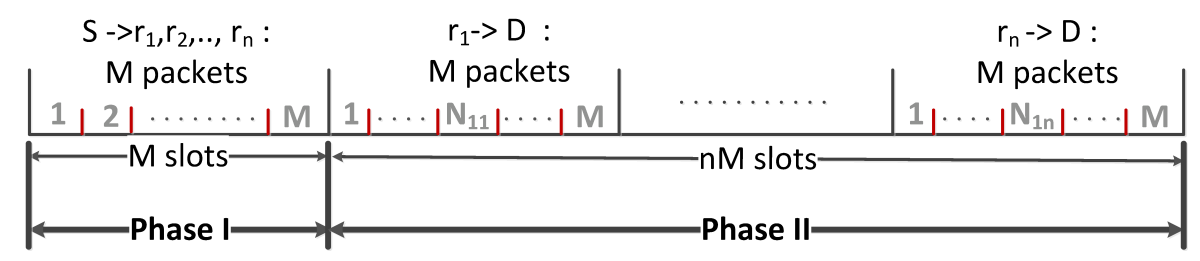

Fig. 2. Time division mechanism for transmitting $M$ recorded measurements from the source to the BNC with the aid of a cluster of relays.

if the packet does not satisfy the QoS requirement, it is dropped. Let $p_{e_{1}}$ be the packet error probability of the $S \rightarrow r_{i}$ wireless links. The $i$-th relay receive $N_{1 i} \leq M$ packets, whose expected number is equal to $\mathbb{E}\left[N_{1 i}\right]=\left(1-p_{e_{1}}\right) M$. In Phase II $\left(P_{I I}\right)$, each relay node is assigned $M$ time slots in order to forward the $N_{1 i}$ received packets to the BNC. FWbased and RLNC-based relaying protocols are considered. In the FW case, the packets are transmitted in a round-robin fashion, i.e., $\bmod \left(M, N_{1 i}\right)$ packets are transmitted $\left\lfloor M / N_{1 i}\right\rfloor+1$ times, whereas the remaining $N_{1 i}-\bmod \left(M, N_{1 i}\right)$ packets are transmitted $\left\lfloor M / N_{1 i}\right\rfloor$ times. The motivation of this choice is that the packets are linear combinations of the original information and that they equally contribute to the signal reconstruction. In the RLNC case, on the other hand, an intra-session encoding is applied, i.e., each relay $i$ transmits $M$ random linear combinations of the $N_{1 i}$ received packets. The number of packets received at the body node coordinator $(D)$ from relay $i$ is denoted by $N_{i 2} \leq M$. Let $p_{e_{2}}$ be the packet error probability on the $r_{i} \rightarrow D$ links. The, the expected number of packets received at $D$ from relay $i$ is equal to $\mathbb{E}\left[N_{i 2}\right]=\left(1-p_{e_{2}}\right) M$.

To evaluate and compare FW and RLNC protocols, we use the energy efficiency $(E E)$ metric defined as follows:

$$
E E=\frac{\text { Number of Reconstructed Measurements }}{\text { Total Energy }}=\frac{N l_{m}}{E_{P_{I}}+E_{P_{I I}}} \text { bits/Joule, }
$$

where $E_{P_{I}}, E_{P_{I I}}$ correspond to the average energy consumption during $P_{I}$ and $P_{I I}$, respectively, $N$ is the number of original samples at the source and $l_{m}$ denotes the size of each measurement in bits. The average energy consumption $E_{P_{I}}, E_{P_{I I}}$ can be formulated as 
follows ${ }^{1}$ :

$$
E_{P_{I}}=M^{*}\left(P_{T}+n P_{R}\right) T_{s}, E_{P_{I I}}=n M^{*}\left(P_{T}+P_{R}\right) T_{s}
$$

where $P_{T}$ and $P_{R}$ are the transmit and the receive power, respectively, and $T_{s}$ is the time required for the transmission of one packet of length $l_{p}$, including payload and headers. Moreover, $M^{*}$ denotes the number of packets that guarantees an acceptable reconstruction of the original data at the BNC. This number depends on the relaying protocol (FW/RLNC), the number of available relays in the network $(n)$ and the CS-based reconstruction algorithm. In the following sections, the impact of these three parameters is explicitly investigated.

\section{Relaying Protocols}

In this section, we study the energy efficiency gains of FW-based and RLNC-based relaying protocols. In both cases, in the first phase $\left(P_{I}\right)$ the source transmits $M$ random linear combinations and the received measurements at the relay $r_{i}$ can be formulated as:

$$
\mathbf{y}_{r_{i}}=\mathbf{I}_{e_{1 i}} \mathbf{y}=\mathbf{I}_{e_{1 i}} \mathbf{A}_{s} \mathbf{x}+\mathbf{I}_{e_{1 i}} \mathbf{z}
$$

where $\mathbf{I}_{e_{1 i}}$ is an $N_{1 i} \times M$ matrix that is used to model the packet losses in the $S \rightarrow r_{i}$ link during $P_{I}$. This matrix can be directly derived from the identity matrix $\mathbf{I}_{M}$ after selecting the $N_{1 i}$ rows that correspond to the packets that are correctly received at $D$.

$F W$-Based Cooperation: In the FW case, the relay $r_{i}$ forwards $M$ packets by cyclic repeating the $N_{1 i}$ received packets. In mathematical terms, this can be formulated as follows:

$$
\mathbf{x}_{F W_{i}}=\mathbf{P}_{r_{i}} \mathbf{y}_{r_{i}}=\mathbf{P}_{r_{i}} \mathbf{I}_{e_{1 i}} \mathbf{A}_{s} \mathbf{x}+\mathbf{z}_{F W_{i}}
$$

where $\mathbf{P}_{r_{i}}$ is an $M \times N_{1 i}$ matrix that performs cyclic repetitions of the $N_{1 i}$ received packets at relay $r_{i}$ and $\mathbf{z}_{F W_{i}}=\mathbf{P}_{r_{i}} \mathbf{I}_{e_{1 i}} \mathbf{z}$. The received measurements at $D$ from $r_{i}$ can be formulated as follows:

$$
\mathbf{y}_{F W_{i}}=\mathbf{I}_{e_{i 2}} \mathbf{x}_{F W_{i}}=\mathbf{A}_{F W_{i}} \mathbf{x}+\mathbf{z}_{F W_{d_{i}}},
$$

\footnotetext{
${ }^{1}$ The energy consumption overhead that is introduced by the transmission of the encoding coefficients to the BNC, has been neglected since we assume that the same encoding coefficients remain fixed for a number of $L$ CRs, where $L>N$. Experimental results have shown, that this strategy do not affect the performance of the decoding algorithm in the BNC.
} 
where $\mathbf{A}_{F W_{i}}=\mathbf{P}_{D} \mathbf{I}_{e_{i 2}} \mathbf{P}_{r_{i}} \mathbf{I}_{e_{1 i}} \mathbf{A}_{s}$ and $\mathbf{z}_{F W_{i}}=\mathbf{P}_{D} \mathbf{I}_{e_{i 2}} \mathbf{P}_{r_{i}} \mathbf{I}_{e_{1 i}} \mathbf{z}$. Accordingly, $\mathbf{I}_{e_{i 2}}$ is an $N_{i 2} \times M$ matrix derived from the identity matrix $\mathbf{I}_{M}$ after selecting the $N_{i 2}$ rows that correspond to the packets correctly received at $D$. The matrix $\mathbf{P}_{D}$ is used to select the non-identical rows and entries of $\mathbf{I}_{e_{i 2}} \mathbf{P}_{r_{i}} \mathbf{I}_{e_{1 i}} \mathbf{A}_{s}$ and $\mathbf{I}_{e_{i 2}} \mathbf{P}_{r_{i}} \mathbf{I}_{e_{1 i}} \mathbf{z}$, respectively.

RLNC-Based Cooperation: In this case, the relay $r_{i}$ applies RLNC to the received packets. In mathematical terms, this can be formulated as follows:

$$
\mathbf{x}_{R L N C_{i}}=\mathbf{A}_{r_{i}} \mathbf{y}_{r_{i}}=\mathbf{A}_{r_{i}} \mathbf{I}_{e_{1 i}} \mathbf{A}_{s} \mathbf{x}+\mathbf{A}_{r} \mathbf{I}_{e_{1 i}} \mathbf{z},
$$

where $\mathbf{A}_{r_{i}}$ is an $M \times N_{1 i}$ matrix with Rademacher i.i.d. coefficients. Thus, the received packets at $D$ can be formulated as follows:

$$
\mathbf{y}_{R L N C_{i}}=\mathbf{I}_{e_{i 2}} \mathbf{x}_{R L N C_{i}}=\mathbf{A}_{R L N C_{i}} \mathbf{x}+\mathbf{z}_{R L N C_{d_{i}}},
$$

with $\mathbf{A}_{R L N C_{i}}=\mathbf{I}_{e_{i 2}} \mathbf{A}_{r_{i}} \mathbf{I}_{e_{1 i}} \mathbf{A}_{s}$ and $\mathbf{z}_{R L N C_{d_{i}}}=\mathbf{I}_{e_{i 2}} \mathbf{A}_{r_{i}} \mathbf{I}_{e_{1 i}} \mathbf{z}$. In the next two sections, we compare the two relaying protocols in single and multi-relay networks. First, we focus our attention on the single relay case $(n=1)$ and, then, the analysis is generalized for application to an arbitrary number $n$ of cooperative relays.

\section{A. Single-Relay Case}

Assume that $r_{1}$ is the unique relay in the network. The vector of the received packets at $D$, both for FW-based (eq. (13)) and RLNC-based (eq. (15)) relaying can be formulated as $\mathbf{y}$ $=\mathrm{Ax}+\mathrm{z}$. Consequently, the original signal $\mathbf{x}$ can be reconstructed at $D$ by using any of the algorithms presented in eqs. (1) and (5). As a result, the energy efficiency potential of both cooperative schemes is directly linked to the number of encoded $M^{*}$ that should be transmitted from the source in order to ensure an accurate reconstruction at $D$. More

specifically, the energy efficiency gain of RLNC-based cooperation compared to FW-based cooperation is quantified by the following proposition.

Proposition 1: In single-relay networks (i.e, $n=1$ ), independently of the CS-based reconstruction algorithm, the energy efficiency gain of RLNC-based cooperation against FW-based cooperation can be formulated as follows:

$$
\frac{E E_{R L N C}}{E E_{F W}}=\frac{\min \left(\left(1-p_{e_{1}}\right),\left(1-p_{e_{2}}\right)\right)}{a\left(1-p_{e_{2}}^{b+1}\right)+\left(\left(1-p_{e_{1}}\right)-a\right)\left(1-p_{e_{2}}^{b}\right)}
$$


where $a=\bmod \left(1,\left(1-p_{e_{1}}\right)\right), b=\left\lfloor 1 /\left(1-p_{e_{1}}\right)\right\rfloor$ and $p_{e_{1}}, p_{e_{2}}$ are the packet error probabilities on the $S \rightarrow r_{1}$ and $r_{1} \rightarrow D$ links, respectively.

Proof: See the Appendix.

By direct inspection of eq. (16), it follows that $\frac{E E_{R L N C}}{E E_{F W}} \rightarrow 1$ as $p_{e_{i}} \rightarrow 0, \forall i$. This implies that RLNC-based cooperation does not introduce any gain compared to the FW-based cooperation under the ideal operating scenario of perfect channels with no link failures. On the other hand, as the error probabilities $p_{e_{1}}$ and $p_{e_{2}}$ increase, the gain provided by RLNCbased cooperation increases. In addition, it can be verified that this gain is maximized in the symmetric case, i.e., if $p_{e_{1}}=p_{e_{2}}$. These performance trends are substantiated in Section VI with the aid of numerical simulations based on real ECG and EEG data.

\section{B. Multi-Relay Case}

In the multi-relay case, we assume that $n$ cooperative relays are available in the network and operate according to the communication protocol described in Section III. As far as the FW-based protocol is concerned, the received packets $\mathbf{y}_{F W}=\left[\mathbf{y}_{F W_{1}}^{T}, \ldots, \mathbf{y}_{F W_{n}}^{T}\right]^{T}$ can be formulated as follows:

$$
\mathbf{y}_{F W}=\mathbf{A}_{F W} \mathbf{x}+\mathbf{z}_{F W},
$$

where $\mathbf{A}_{F W}=\left[\mathbf{A}_{F W_{1}}^{T}, \ldots, \mathbf{A}_{F W_{n}}^{T}\right]^{T}, \mathbf{A}_{F W_{i}}=\mathbf{I}_{e_{i 2}} \mathbf{P}_{r} \mathbf{I}_{e_{i 1}} \mathbf{A}_{s}$ and the noise vector is given by $\mathbf{z}_{F W}=\left[\mathbf{z}_{F W_{d_{1}}}, \ldots, \mathbf{z}_{F W_{d_{n}}}\right]$. In the case of RLNC-based cooperation, the received packets $\mathbf{y}_{R L N C}=\left[\mathbf{y}_{R L N C_{1}}^{T}, \ldots, \mathbf{y}_{R L N C_{n}}^{T}\right]^{T}$ can be formulated as follows:

$$
\mathbf{y}_{R L N C}=\mathbf{A}_{R L N C} \mathbf{x}+\mathbf{z}_{R L N C}
$$

where $\mathbf{A}_{R L N C}=\left[\mathbf{A}_{R L N C_{1}}^{T}, \ldots, \mathbf{A}_{R L N C_{n}}^{T}\right]^{T}, \mathbf{A}_{R L N C_{i}}=\mathbf{I}_{e_{i 2}} \mathbf{A}_{r_{i}} \mathbf{I}_{e_{i 1}} \mathbf{A}_{s}$ and $\mathbf{z}_{R L N C}=\left[\mathbf{z}_{R L N C_{d_{1}}}\right.$, $\left.\ldots, \mathbf{z}_{R L N C_{d_{n}}}\right]$.

The energy efficiency potential of FW-based and RLNC-based cooperation, in the multirelay case, is summarized in the following proposition.

Proposition 2: In multi-relay networks (i.e, $n>1$ ), independently of the CS-based reconstruction algorithm, the energy efficiency gain of RLNC-based cooperation compared to FW-based cooperation can be formulated as follows:

$$
\frac{E E_{R L N C}}{E E_{F W}}=\frac{\min \left(\left(1-p_{e_{1}}^{n}\right), n\left(1-p_{e_{2}}\right)\right)}{a\left(1-p_{e_{2}}^{n(b+1)}\right)+\left(\left(1-p_{e_{1}}^{n}\right)-a\right)\left(1-p_{e_{2}}^{n b}\right)}
$$


where $a=\bmod \left(1,\left(1-p_{e_{1}}^{n}\right)\right), b=\left\lfloor 1 /\left(1-p_{e_{1}}^{n}\right)\right\rfloor$ and $p_{e_{1}}, p_{e_{2}}$ are the packet error probability on the $S \rightarrow r_{i}$ and $r_{i} \rightarrow D$ for $i=1, \ldots, n$ links, respectively.

Proof: See the Appendix.

Since $p_{e_{i}}<1$ by definition, it follows that $p_{e_{i}}^{n}$ tends to zero as $n$ tends to infinity. By direct inspection of eq. (19), this implies that $E E_{R L N C}=E E_{F W}$ if $n$ tends to infinity. As a result, in application scenarios where the number of available relays is very large, RLCN-based cooperation does not provide any gains compared to FW-based cooperation. However, in typical and realistic scenarios where $n$ is relatively small (e.g., $n \leq 3$ ), the numerical examples shown in Section VI, confirm the superiority of RLNC compared to FW.

\section{Efficient CS-Based Signal Reconstruction}

Let $\mathbf{y}=\mathbf{A x}+\mathbf{z}$ be the vector of received packets at the destination. Given the vector $\mathbf{y}$ and the encoding coefficients $\mathbf{A}$, the algorithms presented in Sections II.A constitute the most common options for reconstructing $\mathbf{x}$ at the $\mathrm{BNC}(D)$. In this section, we introduce DIG LASSO, which is a more efficient CS-based reconstruction algorithm that exploits block sparsity properties of a decorrelated version of the original signal, either in the time or in a transform domain, as discussed in Sections II.B and II.C. In Section VI, we show that the DIG LASSO allows the reconstruction of biosignals by using fewer measurements compared to the conventional LASSO, thus potentially enhancing the energy efficiency and the lifetime of the sensor nodes.

\section{A. Temporal Correlation-Aware Block Sparse Recovery}

In [28] and [29], it is shown that the exploitation of a block-sparse structure enables signal recovery from a reduced number of random linearly encoded measurements. Furthermore, in [14] it is proved that biosignals are often block-sparse in different domains. Motivated by these papers, we assume that the signal of interest can be divided in clusters of length $d$, as shown in eq. (2). However, none of the considered biosignals and their representation in a transform domain are strictly block sparse (i.e., consist of blocks of zeros). For evaluating the block compressibility of these signals, we use the so-called $k$ block-term approximation error [24], which is a metric that quantifies the accuracy of their associated block-sparse 
representation. More specifically, let $\mathbf{x}$, where $I=\left[i_{1}, \ldots, i_{R}\right]$ denotes the signal that consists of the blocks of $\mathbf{x}$ that are ordered according to their $l_{2}$ norm, e.g., $\mathbf{x}_{I}=\left[\mathbf{x}^{T}\left[i_{1}\right], \ldots, \mathbf{x}^{T}\left[i_{R}\right]\right]$, where $\mathbf{x}\left[i_{j}\right]=\left[\left(i_{j}-1\right) d+1, \ldots, i_{j} d\right], j=1, \ldots, R$ and

$$
\left\|\mathbf{x}\left[i_{1}\right]\right\|_{2} \geq\left\|\mathbf{x}\left[i_{2}\right]\right\|_{2} \geq\left\|\mathbf{x}\left[i_{3}\right]\right\|_{2} \geq \ldots \geq\left\|\mathbf{x}\left[i_{R}\right]\right\|_{2}
$$

Let $\mathbf{x}_{k}, 1 \leq k \leq R$ denote the $k$-term approximation of $\mathbf{x}$, which is obtained by setting to zero the blocks from $i_{k+1}$ to $i_{R}$, i.e., $\mathbf{x}_{k}=\left[\mathbf{x}^{T}\left[i_{1}\right], \ldots, \mathbf{x}^{T}\left[i_{k}\right], \mathbf{0}_{d}^{T}, \ldots, \mathbf{0}_{d}^{T}\right]$. Let the vectors $\mathbf{x}_{I}$ and $\mathbf{x}_{k}$, the mean $k$ block-term approximation is defined as follows:

$$
\overline{e_{k}}=E\left[\frac{\left\|\mathbf{x}_{I}-\mathbf{x}_{k}\right\|_{2}}{\left\|\mathbf{x}_{I}\right\|_{2}}\right]
$$

Several sparse representation methods, such as the discrete cosine/wavelet transfrom (DCT, DWT) or the Principal Component Analysis (PCA) transform [30], have been used in the past for enhancing the sparseness of biosignals [7], [31]. Their sparse representation efficiency is based on the fact that they tend to decorrelate (e.g., DCT, DWT) or they completely decorrelate (e.g., PCA) the components of a given signal and redistribute the energy contained in the signal so that most of energy is contained in a small number of components. Motivated by property, we propose a transformation that performs decorrelation of the biosignal samples within a block. In particular, we assume that: i) a positive definite matrix $\mathbf{R}_{d} \in \mathbb{R}^{d \times d}$ captures the correlation structure of the $i$-th block of recorded measurements $\mathbf{x}[i]$ and ii) the correlation between elements of different signal blocks is almost zero, since these signals can be approximated by using block sparse representations that consist of zero and non-zero blocks. To model the correlation among the samples of the same block, we assume that the signal samples within a block can be modeled as an auto regressive - 1 process (AR-1) [6], [32 ${ }^{2}$. Accordingly, the correlation matrix, $\mathbf{R}_{d}$, of each block can be written as a Toeplitz symmetric matrix with elements $\mathbf{R}_{d i, j}=\rho^{|i-j|}, \forall i, j \in[1, \ldots, d]$ and the decorrelation of signal $\mathbf{x}$ can be performed as follows:

$$
\mathbf{s}=\mathbf{R}^{-1 / 2} \mathbf{x}, \mathbf{R}^{-1 / 2}=\left[\begin{array}{cccc}
\mathbf{R}_{d}^{-1 / 2} & \mathbf{0}_{d} & \ldots & \mathbf{0}_{d} \\
\ldots & \ddots & \ddots & \ldots \\
\mathbf{0}_{d} & \ldots & \ldots & \mathbf{R}_{d}^{-1 / 2}
\end{array}\right]
$$

\footnotetext{
${ }^{2}$ Higher order models may be used, but AR(1) constitutes a good compromise between complexity and performance.
} 

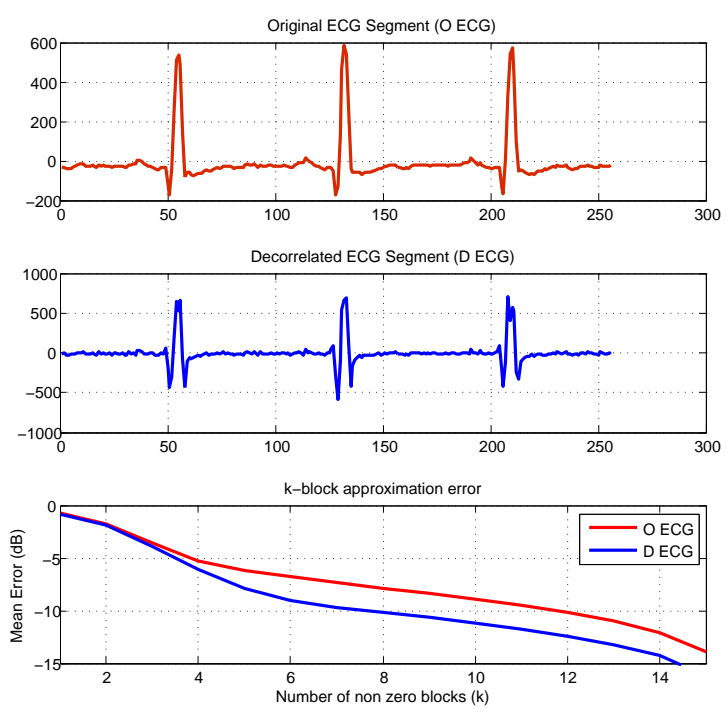

Fig. 3. Original (O) and Decorrelated (D) ECG segment, denoted as O-ECG and D-ECG respectively, which correspond to a 2 seconds recording of a male patient retrieved from the MIT-BIH Normal Sinus Rhythm Database [33]. The $k$ block approximation error, which evaluated over all the ECG segments taken from the MIT-BIH Normal Sinus Rhythm Database [33], is also provided for assessing the compressibility of O-ECG and D-ECG signals.

To evaluate the effectiveness of the proposed transformation, in Fig. 3 we illustrate an example that is obtained by using ECG data taken from the the MIT-BIH Normal Sinus Rhythm Database [33]. The figures shows the k-block term approximation error of the original signal $\mathbf{x}$ and of its decorrelated version $\mathbf{s}$, from which the effectiveness of the proposed approach is apparent. The impact of the parameter $\rho$ of the AR-1 model on the recovery performance of the proposed scheme is evaluated in Section VI.

Motivated by these observations, we propose to take advantage of the block sparsity of the decorrelated version of $\mathbf{x}$. By choosing $\Psi=\mathbf{R}^{1 / 2}$, the problem defined in eq. (7) can be re-formulated as follows:

$$
\hat{\mathbf{s}}:=\underset{\mathbf{s}}{\arg \min }\left\|\mathbf{y}-\mathbf{A R}^{1 / 2} \mathbf{s}\right\|_{2}^{2}+\sum_{i=1}^{R} \lambda_{i}\|\mathbf{s}[i]\|_{2} .
$$

After solving it, the original signal $\mathbf{x}$ can be reconstructed by inverting eq. (22). 
TABLE I

Decorrelated Iterative Reweighted Group (DIG) LASSO Recovery Algorithm

\section{DIG LASSO:}

Inputs: Encoding Matrix: A, Dictionary*: $\Psi$, Received packets: $\mathbf{y}$, Block length $d$, Block Sparsity $k$ Output: Estimated ECG Data $\mathbf{x}$

1. Initialize $\mathbf{x}^{(0)}, w_{i}^{(0)}, \epsilon$

a. Solve the following problem: $\mathbf{x}^{(0)}:=\arg \min _{x}\|\mathbf{y}-\mathbf{A x}\|_{2}^{2}+\lambda \sum_{i=1}^{R}\|\mathbf{x}[i]\|_{2}$

by applying Interior Point (IP) methods

b. Initialize weights: $w_{i}^{(0)}:=\left(\left\|\mathbf{x}^{(0)}[i]\right\|_{2}+\epsilon\right)^{-1}, \quad i=1, \ldots, R$

2. Initialize $\mathbf{s}^{(0)}[i] \forall i \in\{1, \ldots, R\}: \mathbf{s}^{(0)}[i]=\mathbf{R}_{i}^{-1 / 2} \Psi^{-1} \mathbf{x}[i]$

3. Repeat for each iteration $l=1, \ldots, K$

a. Solve problem: $\mathbf{s}^{(l)}:=\arg \min _{s}\left\|\mathbf{y}-\mathbf{A} \Psi \mathbf{R}^{1 / 2} \mathbf{s}\right\|_{2}^{2}+\sum_{i=1}^{R} \lambda_{i} w_{i}^{(l)}\|\mathbf{s}[i]\|_{2}$

by applying IP methods

b. Evaluate $\mathbf{x}^{(l)}[i]=\mathbf{R}_{i}^{1 / 2} \Psi \mathbf{s}^{(l)}[i] \forall i$

c. Update weights $w_{i}^{(l+1)}$ according to eq. (30)

d. Reduce $\epsilon$ by a factor of 10 . end repeat

${ }^{*}$ To execute the algorithm in the time domain, we select $\Psi=\mathbf{I}_{N}$

\section{B. Enhancing Recovery Efficiency by Iterative Reweighting}

In eq. (23), similar to the LASSO algorithm, the non smooth indicator function $I(\mathbf{s}[i] \neq 0)$ is approximated by its closest convex approximation $\|\mathbf{s}[i]\|_{2}$. However, by replacing it with a non-convex function may lead to a tighter approximation, which may further enhance the recovery efficiency of the group LASSO algorithm. In [34], for example, the indicator function $I(|x|=0)$ for $x \in \Re$ bas been replaced with the function $\log (|x|+\epsilon)$, where $\epsilon>0$ is a constant that avoids the singularity at $-\infty$. Building upon this idea, the optimization in (23) can be replaced by the following:

$$
\hat{\mathbf{s}}:=\underset{\mathbf{s}}{\arg \min }\left\|\mathbf{y}-\mathbf{A R}^{1 / 2} \mathbf{s}\right\|_{2}^{2}+\lambda \sum_{i=1}^{R} \log \left(\|\mathbf{s}[i]\|_{2}+\epsilon\right),
$$

which is a non-convex problem whose minimization is non-trivial. The concavity of the logarithm implies that a linearization of $\log \left(\|\mathbf{s}[i]\|_{2}+\epsilon\right)$ around any $\left\|\mathbf{s}^{(0)}[i]\right\|_{2}>0$ can be used as a majorization function, i.e.,

$$
\log \left(\|\mathbf{s}[i]\|_{2}+\epsilon\right) \leq \log \left(\left\|\mathbf{s}^{(0)}[i]\right\|_{2}+\epsilon\right)+\frac{\|\mathbf{s}[i]\|_{2}-\left\|\mathbf{s}^{(0)}[i]\right\|_{2}}{\left\|\mathbf{s}^{(0)}[i]\right\|_{2}+\epsilon} .
$$


Thus, given any $\left\|\mathbf{s}^{(l)}[i]\right\|_{2}$, the cost in (24) can be majorized by the following function:

$$
\left\|\mathbf{y}-\mathbf{A} \mathbf{R}^{1 / 2} \mathbf{s}\right\|_{2}^{2}+\lambda \sum_{i=1}^{R} \frac{\|\mathbf{s}[i]\|_{2}}{\left\|\mathbf{s}^{(l)}[i]\right\|_{2}+\epsilon} .
$$

Following the majorization-minimization approach presented in [35], and letting $\mathbf{s}^{(l)}$ be the estimates at the $l$-th iteration, eq. $((5))$ can be driven to a stationary point by updating $\mathbf{s}^{(l+1)}$ as the minimizer of:

$$
\begin{aligned}
\mathbf{s}^{(l+1)} & :=\underset{s}{\arg \min }\left\|\mathbf{y}-\mathbf{A} \mathbf{R}^{1 / 2} \mathbf{s}\right\|_{2}^{2}+\lambda \sum_{i=1}^{R} w_{i}^{(l)}\|\mathbf{s}[i]\|_{2} \\
w_{i}^{(l)} & :=\left(\left\|\mathbf{s}^{(l)}[i]\right\|_{2}+\epsilon\right)^{-1}, \quad i \in[1, R] .
\end{aligned}
$$

where $\mathbf{s}^{(l+1)}$ denotes the estimate of $\mathbf{s}$ that was evaluated in the $l+1$ iteration. According to the study that was carried out in [36], the strategy of using a relatively large $\epsilon$ in the first iteration and then repeating the process of decreasing $\epsilon$ after each iteration $l$ (gradual reduction strategy - GRS) can be very effective in avoiding suboptimal local minima troubles.

The optimization per iteration of eq. (27) is a weighted version of eq. (7), where $\mathbf{W}=$ $\mathbf{R}^{-1 / 2}$ and, thus, it can be efficiently solved by using standard software packages. The iterations can be initialized with the solution of eq. (7), which corresponds to setting all weights equal to one. The proposed iterative DIG LASSO algorithm is terminated as soon as the relative error $\left\|\mathbf{s}^{(l)}-\mathbf{s}^{(l-1)}\right\|_{2} /\left\|\mathbf{S}^{(l)}\right\|_{2}$ becomes smaller than some predefined small value (e.g., $10^{-6}$ ). it will be shown that for the specific considered application, even one additional iteration (e.g., $K=2$ ) can significantly improve the solution of (7). The original signal after $K$ iterations can be reconstructed as $\hat{\mathbf{x}}=\mathbf{R}^{1 / 2} \mathbf{s}^{(K)}$. The steps of the proposed DIG LASSO algorithm are summarized in Table I, while the benefits of the proposed algorithm in terms of convergence (i.e., required samples for achieving an accurate reconstruction at the receiver) are evaluated in Section VI.

\section{DIG LASSO in a Transform Domain}

Following the same line of thought as that of Section II.C, the DIG LASSO can be also applied in a transform domain, where the transformed signal has similar sparsity characteristics as the original signal in Section II.C. Let us assume that there is a dictionary 
matrix $\Psi$ of dimensions $N \times N$, so that $\mathbf{x}$ can be expressed as $\mathbf{x}=\Psi \mathbf{s}$. From the received measurements at the BNC, it is possible to reconstruct $\mathbf{s}$ by first exploiting its temporal correlations and by then reconstructing $\mathbf{x}$. In mathematical terms, the problem can be iteratively formulated as follows:

$$
\begin{aligned}
\mathbf{s}^{(l)} & :=\underset{s}{\arg \min }\left\|\mathbf{y}-\mathbf{A} \boldsymbol{\Psi} \mathbf{R}^{1 / 2} \mathbf{s}\right\|_{2}^{2}+\lambda \sum_{i=1}^{R} w_{i}^{(l)}\|\mathbf{s}[i]\|_{2} \\
w_{i}^{(l)} & :=\left(\left\|\mathbf{s}^{(l-1)}[i]\right\|_{2}+\epsilon\right)^{-1}, \quad i \in[1, R] .
\end{aligned}
$$

The original signal $\mathbf{x}$ can be reconstructed after $l=K$ iterations as $\hat{\mathbf{x}}=\Psi \mathbf{R}^{1 / 2} \mathbf{S}^{(K)}$.

\section{Performance Evaluation}

In this section, we employ real biosignal data to study the performance of the DIG LASSO reconstruction algorithm and the RLNC-based cooperative protocol. Two case studies are analyzed: i) ECG signals, which are block-sparse in the time domain and ii) EEG signals, which are block-sparse in a transform domain. The proposed solutions are compared against state-of-the-art LASSO reconstruction algorithms and FW-based cooperative protocols in terms of reconstruction accuracy and complexity, as well as energy efficiency and robustness to link failures.

\section{A. Simulation Setup}

We assume that each ECG/EEG signal is divided in segments of $N=128$ samples that correspond to a signal observation of $2 \mathrm{~s}$ duration. Each segment is encoded at the biosensor side by generating $M$ RLNC-encoded packets, which are transmitted to a set of relays. Each relay is capable of applying FW-based or RLNC-based relaying. Transmit and receive powers are equal to $3.8 \mathrm{~mW}$ and $4.6 \mathrm{~mW}$, respectively. We also assume a packet length of 14 bytes with 2 bytes used for the payload, a data rate equal to $256 \mathrm{kbps}$ [4] and $l_{m}=16$ bits/measurement. The duration of a packet transmission is assumed to be $0.44 \mathrm{~ms}$ [37]. With regard to the reconstruction of the signal at the BNC, LASSO and DIG LASSO algorithms, applied in the time domain (ECG case) and in the Discrete Cosine Transform (DCT) domain (EEG case), are studied. As a result, four different telemonitoring schemes are compared: (i) DIG LASSO-RLNC, (ii) LASSO-RLNC, (iii) DIG LASSO-FW and (iv) LASSO-FW. 
TABLE II

ENERGY EFFICIENCY (10 ${ }^{6}$ BITS/JOULE) OF WIRELESS TELEMONITORING SCHEMES CASE STUDY: ECG

\begin{tabular}{|c||c|c|c|c||c|c|c|c|}
\hline \multicolumn{1}{|c||}{} & \multicolumn{3}{c||}{ Single-Relay Case - n=1 } & \multicolumn{3}{c|}{ Multi-Relay Case - n=3 } \\
\cline { 2 - 9 } & DIG LASSO & \multicolumn{2}{c|}{ LASSO } & DIG LASSO & \multicolumn{2}{c|}{ LASSO } \\
\cline { 2 - 9 } & RLNC & FW & RLNC & FW & RLNC & FW & RLNC & FW \\
\hline \hline$p_{e}=0.15$ & 2.88 & 2.50 & 1.69 & 1.46 & 1.32 & 1.31 & 0.77 & 0.77 \\
\hline$p_{e}=0.25$ & 2.54 & 2.05 & 1.48 & 1.20 & 1.30 & 1.28 & 0.76 & 0.75 \\
\hline$p_{e}=0.35$ & 2.18 & 1.68 & 1.28 & 0.98 & 1.26 & 1.21 & 0.74 & 0.71 \\
\hline$p_{e}=0.45$ & 1.84 & 1.39 & 1.09 & 0.81 & 1.20 & 1.10 & 0.70 & 0.65 \\
\hline$p_{e}=0.55$ & 1.51 & 1.11 & 0.89 & 0.64 & 1.10 & 0.95 & 0.65 & 0.58 \\
\hline$p_{e}=0.65$ & 1.17 & 0.82 & 0.69 & 0.47 & 0.96 & 0.77 & 0.56 & 0.45 \\
\hline
\end{tabular}

In each experiment, all schemes are evaluated by using the same sensing matrices $\left(\mathbf{A}, \mathbf{A}_{r}\right)$, either for performing RLNC of the ECG/EEG encoding at the biosensor or for performing RLNC of the packets received at the relay.

\section{B. ECG Case Study}

1) ECG Dataset and Performance Metrics: The empirical data for the evaluation of the proposed signal processing algorithm and communication protocol have been retrieved from the MIT-BIH Normal Sinus Rhythm Database [33], which includes 18 long-term ECG recordings of subjects referred to the Arrhythmia Laboratory at Boston's Beth Israel Hospital (now the Beth Israel Deaconess Medical Center). The dataset is referred to 5 men, aged between 26 and 45, and to 13 women, aged between 20 and 50. The recordings are digitized at 128 samples per second per channel.

In order to evaluate the diagnostic quality of the reconstructed ECG recordings at the BNC, we employ the Percentage Root-mean-square Difference (PRD) [38] metric, which is defined as: $P R D=\|\mathbf{x}-(\tilde{\mathbf{x}})\|_{2} /\|\mathbf{x}\|_{2} \times 100$, where $\mathbf{x}$ and $\tilde{\mathbf{x}}$ denote the original and the reconstructed signal, respectively. Similar to [38], we assume that a reconstructed signal is acceptable at the BNC only if $P R D<9 \%$ [38].

2) Validation of Propositions 1 and 2: To validate Proposition 1 and 2, we have conducted simulation experiments for single and multi-relay scenarios, aiming at evaluating the energy efficiency gains of RLNC compared to FW relaying. The reconstruction of the ECG segments 


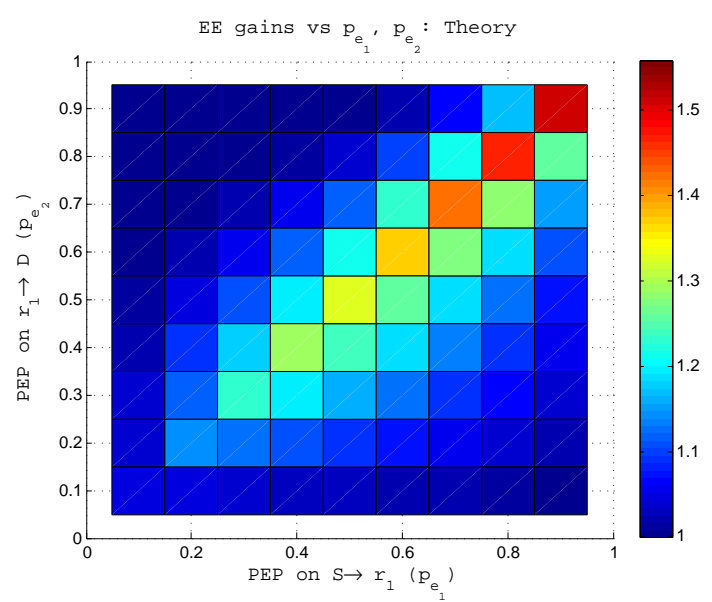

(a)

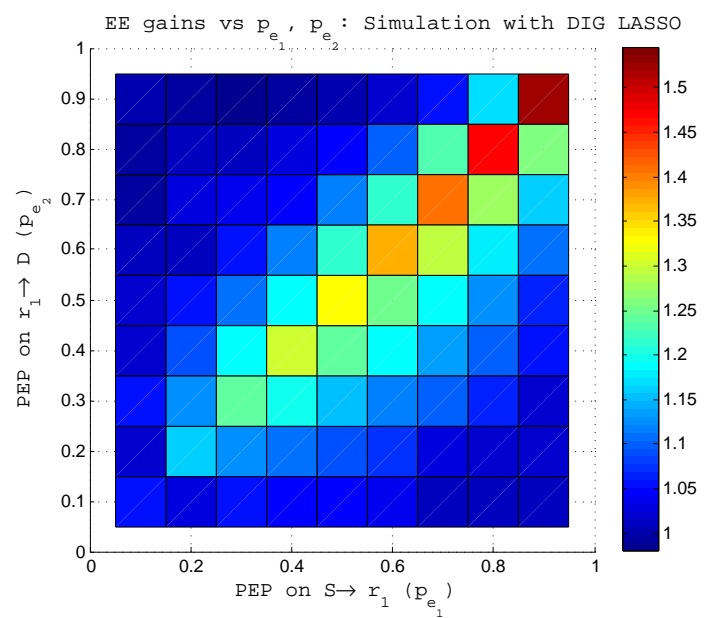

(c)

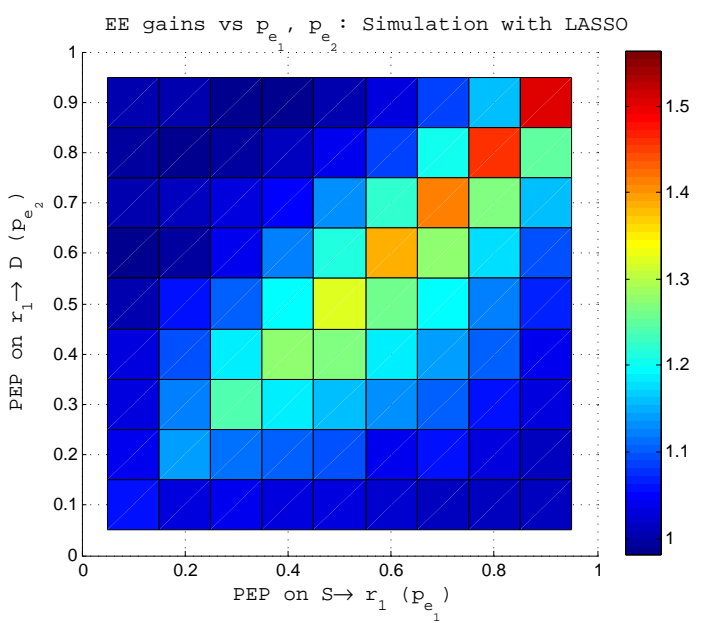

(b)

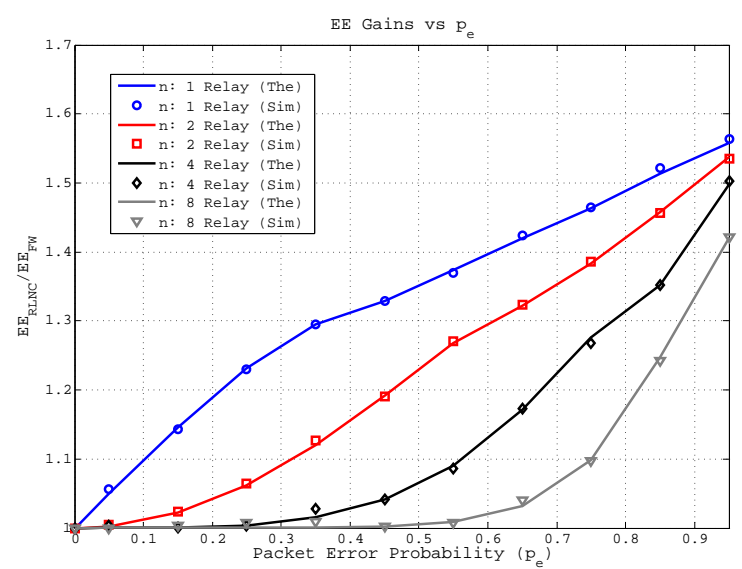

(d)

Fig. 4. Validation of Proposition 1 and 2. Energy efficiency gains of the RLNC compared to the FW case for different values of the PEP at the links $S \rightarrow r_{i}$ and $r_{i} \rightarrow D:(a)$ Theoretical Results for single relay case (b) Simulation Results for single relay case by executing LASSO at the BNC (c) Simulation Results for single relay case by executing DIG LASSO at the BNC (c) Theory vs Simulation for different number of Relays, assuming that $p_{e_{1}}=p_{e_{2}}=p_{e}$.

at the BNC is performed by executing the LASSO and the DIG LASSO algorithms. The scaling rules for the parameter $\lambda$ in eqs. (1) and (24) are chosen by solving (1) and (24) for a grid of $\lambda$ values and selecting those that minimize the number of transmit packets for ensuring an accurate reconstruction (e.g., $\operatorname{Pr}\{P R D<9 \%\}>0.98$ ) at the BNC. As for the DIG LASSO algorithm, a block length $d=16$ is adopted. Iterations of the DIG LASSO 


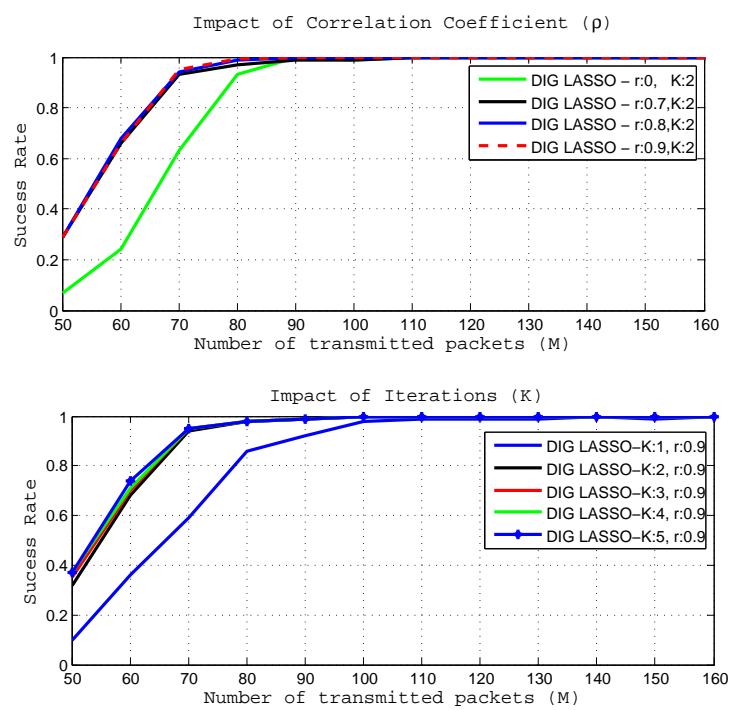

(a)

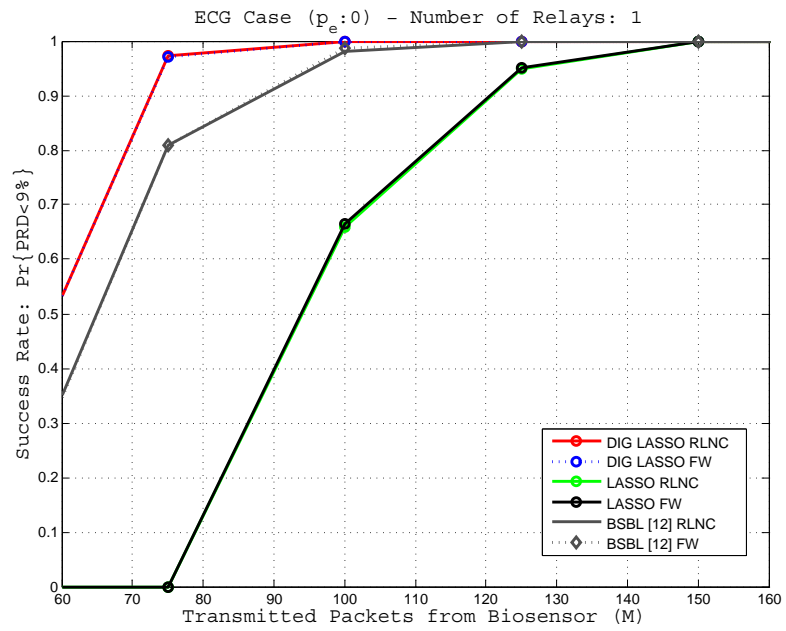

(b)

Fig. 5. (a) Effects of the correlation coefficient $\rho$ and the number of iterations $K$ to the DIG LASSO Success Rate (b) Evaluation of different recovery algorithms in terms of the average PRD assuming an error probability $p_{e}=0$.

scheme are initialized to the solution of (23), while the maximum number of iterations is equal to $K=3$. The success rate $(\operatorname{Pr}\{P R D<9 \%\})$ has been evaluated by using 1 h of 18 different ECG segments of the MIT-BIH Normal Sinus Rhythm Database [33]. In Fig. 4(a), we present the theoretical gains in the single relay case for different values of the packet error probabilities $p_{e_{1}}$ and $p_{e_{2}}$ by applying eq. (16). Figure 4(b) and (c) present the ratio $E E_{R L N C} / E E_{F W}$, after executing the LASSO and the DIG LASSO schemes, respectively. In Fig. 4(d), we plot theoretical and simulation results for single and multi-relay scenarios, by assuming equal error probabilities for the $S \rightarrow r_{i}$ and $r_{i} \rightarrow D$ links.

The figures confirm the accuracy of the proposed mathematical frameworks. Moreover, they demonstrate that the energy efficiency gain of RLNC increases as the packet error probabilities $p_{e_{1}}$ and $p_{e_{2}}$ increase, and this gain is maximized $p_{e_{1}}=p_{e_{2}}$. On the other hand, as the number of relays increases, the gain decreases. Similar conclusions can be drawn by inspecting Table II, where the network energy efficiency based on eqs. (9)-(10) is reported for different values of $p_{e}$ and for single $(n=1)$ and multi-relay $(n=3)$ scenarios. In the multi-relay case, the results show that the use of relays: a) is beneficial for the source, since it reduces the number of packets to be transmitted in the network for 


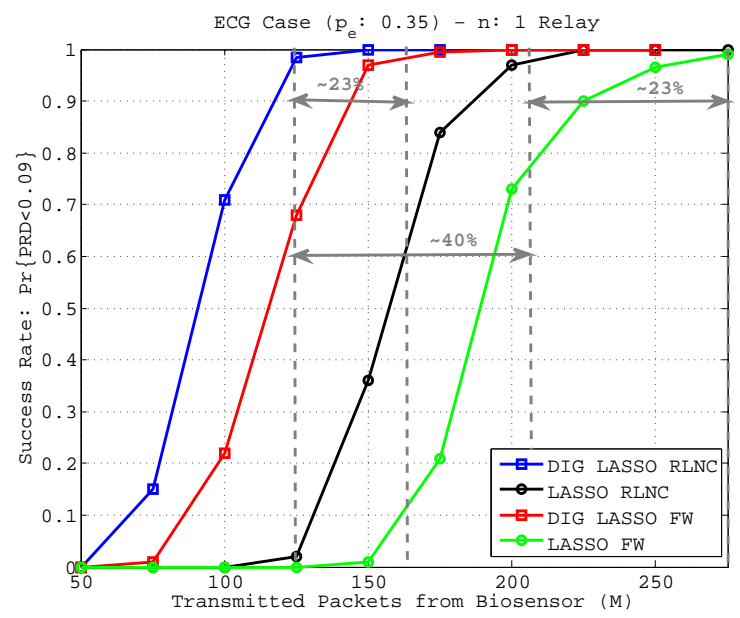

(a)

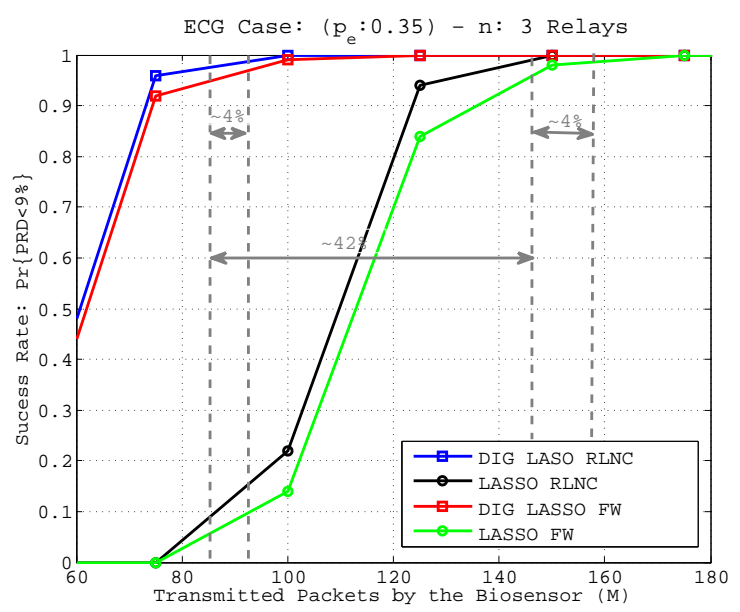

(c)

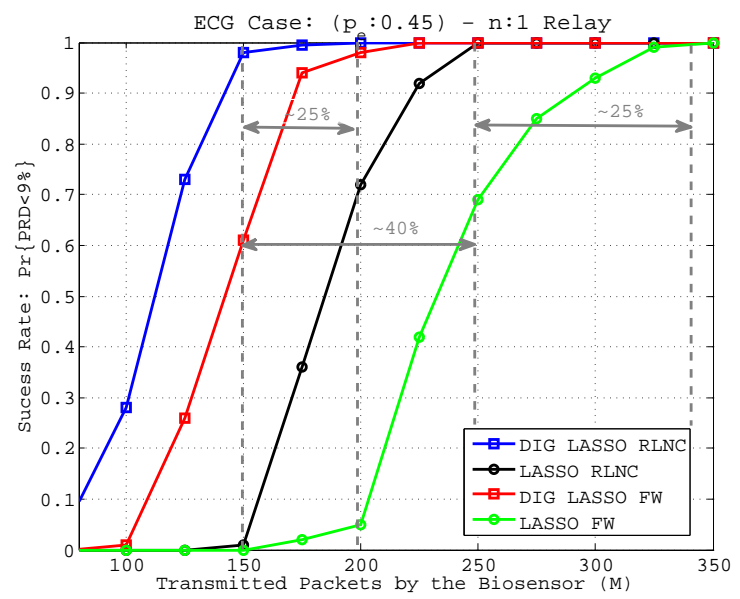

(b)

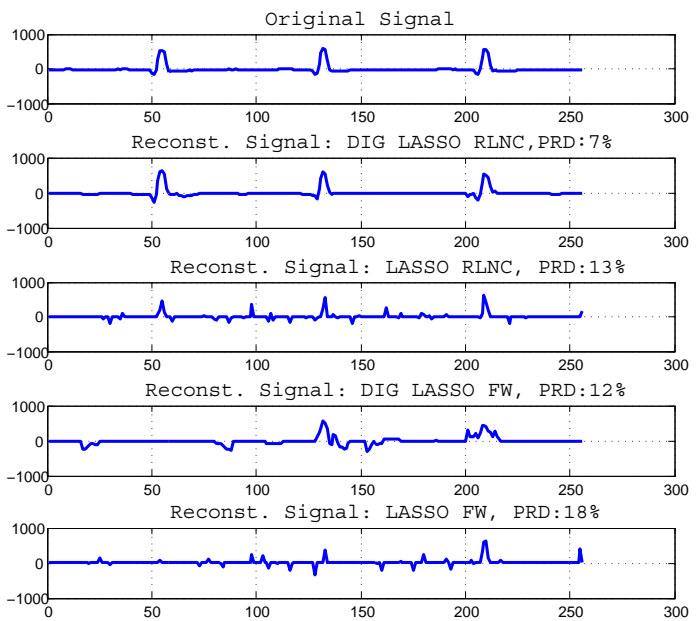

(d)

Fig. 6. Evaluation of different telemonitoring schemes in terms of (a) success rate for $p_{e}=0.35$ and $n=1$, (b) success rate for $p_{e}=0.45$ and $n=1$, (c) success rate for $p_{e}=0.35$ and $n=3$, (d) Reconstructed signals using the evaluated telemonitoring schemes for $M=120$ and $p_{e}=0.4$

ensuring an accurate signal reconstruction at the BNC and b) reduces the network energy efficiency. Based on these results and on the fact that the number of potential cooperative body nodes in real WBANs is limited, it is possible to conclude that RLNC constitutes a promising cooperative relaying protocol for application in WBANs.

3) Performance Evaluation: Having validated the propositions, in this section we focus on the effect of the different parameters and the performance evaluation of the proposed schemes. To that end, we assume a symmetric topology with $p_{e_{1}}=p_{e_{2}}=p_{e}$. In Fig. 5a, we 
examine the impact of i) the correlation coefficient $\rho$ captures the correlation between adjacent samples of a block, and ii) the number of iterations $(K)$ executed during the iterative re-weighting approach on the recovery performance of the DIG LASSO algorithm. More specifically, Fig. 5a provides the success rate of the DIG LASSO-RLNC scheme versus the number of transmitted packets $(M)$ for different values of $\rho$ and $K$, assuming one relay and ideal channel conditions (i.e., $p_{e}=0$ ). By inspecting this figure, we conclude that any values between $0.7 \leq \rho \leq 0.9$ can lead to significant gains compared to the case that exploits the block sparsity in the time domain $(\rho=0)$. Regarding the impact of iterations, we can observe that even one additional iteration of DIG LASSO $(K=2)$ can significantly improve the recovery performance of (23) over (24) and as a result the success rate as well.

By configuring the parameters of DIG LASSO based on the aforementioned study, we have conducted comparisons with the traditional LASSO scheme that was adopted in [3] and with a very recent state-of-the-art CS-based algorithm, which has been applied to ECG signal telemonitoring, i.e., the BSBL algorithm [14]. To that end, in Fig. 5b, we provide the success rate as a function of the number of the transmitted packets $(M)$, assuming one relay and ideal channel conditions (i.e., $p_{e}=0$ ). By inspecting this figure, it can be observed that DIG LASSO achieves an accurate reconstruction with 73 packets, significantly outperforming the LASSO and the BSBL algorithms, which require 140 and 108 packets, respectively. Again, it is shown that, in the ideal case without link failures, the RLNC-based cooperation provides no gain compared to FW-based cooperation, thus validating Proposition 1.

In Fig. 6(a), the success rate as a function of the number of packets $M$ transmitted by the biosensor is plotted. The required number of packets $M^{*}$ that ensures an accurate reconstruction in each case study (i)-(iv) are those that result in a success rate larger than 0.98. In particular, it is shown that the DIG LASSO RLNC (i) leads to an accurate reconstruction by transmitting only $M_{(i)}^{*} \approx 126$ packets, whereas the DIG LASSO FW (iii) requires $M_{(i i i)}^{*} \approx 164$ packets. Similarly, the LASSO RLNC (ii) requires $M_{(i i)}^{*} \approx 215$, whereas at least $M_{(i v)}^{*} \approx 278$ packets are needed by the LASSO FW (iv). Since $M_{(i)}^{*} / M_{(i i i)}^{*} \approx M_{(i i)}^{*} / M_{(i v)}^{*} \approx 0.76$, we conclude that the RLNC-based cooperation results in a $24 \%$ reduction of the total packets required to be transmitted in the network compared to the FW-based cooperation. These findings are also in agreement with Proposition 1. In addition, it can be observed that the DIG LASSO algorithm can achieve accurate reconstruction with $40 \%$ less data than the 
LASSO approach. In Fig. 6(b), the success rate as a function of the number of packets $M$ transmitted by the biosensor for $p_{e}=0.45$ is shown. Again, we observe that the gain offered by RLNC compared to FW is slightly higher compared to the previous case, as it is also stated in Proposition 1. In Fig. 6(d), the reconstructed ECG signal segments that correspond to a $2 \mathrm{~s}$ ECG recording are illustrated, assuming $M=120$ and $p_{e}=0.4$. By inspecting the reconstructed signal, it can be noted that the DIG LASSO RLNC is the only algorithm that provides an acceptable ECG signal reconstruction. As a result, we conclude that the gain of the RLNC policy as compared to the conventional FW scheme increases significantly with $p_{e}$, due to the fact that the RLNC policy ensures the reception of more coded samples at the destination.

In Fig. 6(c), we study the performance of the four schemes in a multi-relay network (i.e., $n=3$ ), assuming $p_{e}=0.35$. In this case, we may notice that the DIG LASSO RLNC requires the transmission of a significantly lower number of packets (i.e., $\sim 86$ packets) from $S$ for an accurate reconstruction compared to the single-relay case (Fig. 6(a)), where approximately 126 packets are needed. Regarding the comparison of the two relaying policies, we can see that, as the number of relays increases in the network, the two schemes tend to achieve similar performance, since the packet loss probability in the multi-relay case is lower than the respective probability in the single-relay scenario. This result is in agreement with Proposition 2, while similar conclusions are also drawn in the LASSO case. By inspecting the reconstructed signal, it can be noted that the DIG LASSO RLNC is the only algorithm that provides an accurate and acceptable ECG signal reconstruction. Note that in all the aforementioned results, the DIG LASSO RLNC reduces the number of packet transmissions by $40 \%$ compared to the LASSO RLNC.

\section{EEG Case Study}

1) EEG Dataset and Performance Metrics: The empirical data for the evaluation of the proposed signal processing algorithms and communication protocol are retrieved from [39]. This database consists of surface EEG recordings from healthy volunteers and intracranial EEG recordings from epilepsy patients. The performance metric used to measure the recovery quality is the Normalized Mean Square Error (NMSE), which is defined as

$1 / 1000 \sum_{i=1}^{1000}\left\|\mathbf{x}-\hat{\mathbf{x}}_{\mathbf{i}}\right\|_{2}^{2} /\|\mathbf{x}\|_{2}^{2}$, where $\hat{\mathbf{x}}_{\mathbf{i}}$ is the estimate of the original signal $\mathbf{x}$ at the $i$-th 


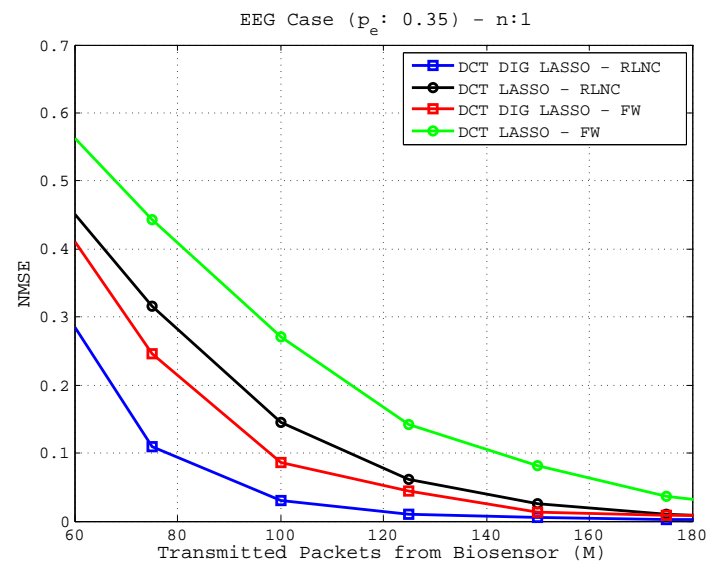

(a)

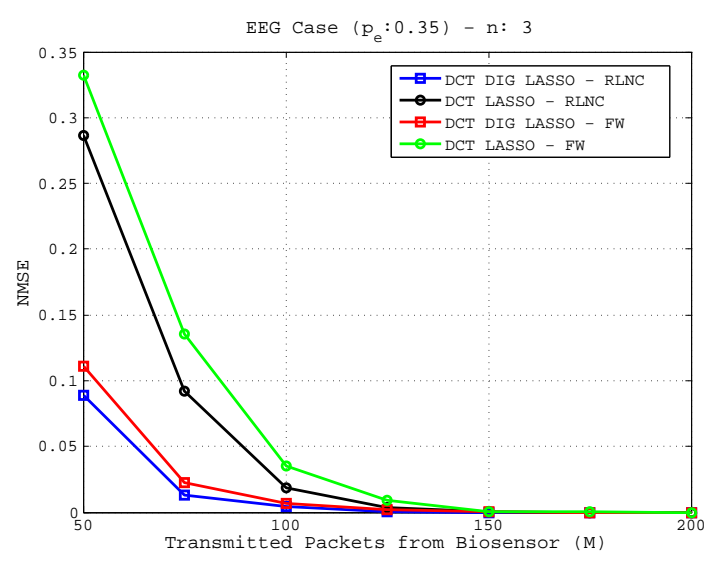

(c)

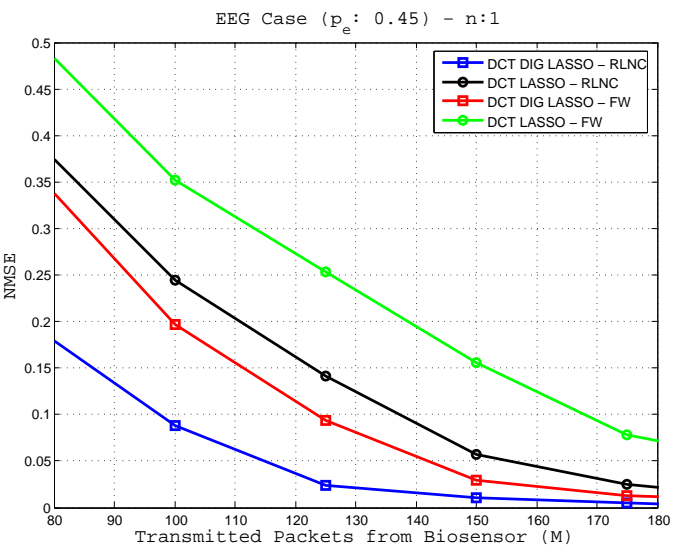

(b)

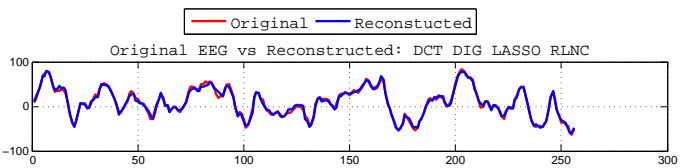

Original EEG vs Reconstructed: DCT LASSO RLNC
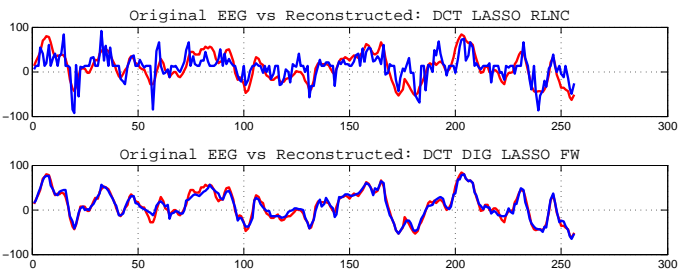

Original EEG vs Reconstructed: DCT LASSO FW

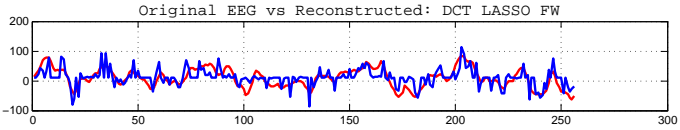

(d)

Fig. 7. Evaluation of different telemonitoring schemes in terms of average PRD. (a) $p_{e}=0.25$, (b) $p_{e}=0.35$, (c) $p_{e}=0.45$. (d) Reconstructed signals for $M=120$ and $p_{e}=0.45$.

Monte Carlo iteration.

2) Performance Results: In Figs.7(a)-(c), the NMSE, averaged over 100 different EEG time series of 4096 samples, is plotted against the number of transmitted packets $(M)$, for different packet error probabilities and number of relays. A block length of $d=16$ is considered and the parameter that determines the amount of correlation between adjacent samples is assumed to be equal to 0.5. These values are chosen in order to ensure the small difference between the norms of non-zero blocks. The figures show that RLNC-based cooperation is more robust to link failures than FW-based cooperation. In addition, the exploitation of temporal correlation and block sparsity in the DCT domain significantly 
reduces the number of packets required for signal reconstruction. The energy efficiency gains can be evaluated following a similar approach as for the ECG case study, but are not reported here due to space limitations. Finally, Fig. 7(d) shows the reconstructed EEG signal segment that corresponds to an EEG recording of $1.5 \mathrm{~s}$ duration. The number of transmitted packets is $M=120$ and a packet error $p_{e}=0.3$ is considered. The results confirm that the DCT DIG LASSO RLNC provides the most accurate reconstruction.

\section{Concluding Remarks}

Real-time vital signal telemonitoring in WBANs is expected to become pervasive in the following years. However, several research challenges need to be addressed for its widespread use and deployment, which include: a) minimizing the amount of data to be transmitted and b) increasing the robustness to link failures. In this paper, we have introduced a novel CCS framework that offers significant gains in terms of energy efficiency and robustness to link failures, by exploiting the benefits of RLNC-based cooperation and CS-based signal processing algorithms that take into account the inherent block sparse structure of several biosignals. We provided closed form expressions for evaluating the RLNC energy efficiency gains as compared to the FW approach, in both single- and multi-relay cases that confirmed the significant gains offered by RLNC, especially in practical scenarios with limited number of relays. The proposed solutions have been tested by using real ECG and EEG data available in open-access medical databases and the numerical examples have validated the expected gains, in terms of enhanced signal reconstruction, robustness to link failures and reduced energy consumption. Similar algorithms and protocols can be applied to other telemedicine applications, such as EMG [3].

\section{APPENDIX}

\section{A. Lemma 1}

Let $X_{1}$ and $X_{2}$ be two mutually independent Rademacher RVs, i.e., $X_{1}= \pm 1 / \sqrt{N}$ and $X_{2}= \pm 1 / \sqrt{M}$ with probability equal to 0.5 . Then, the product $X_{1} X_{2}$ is a subgaussian RV, i.e., $X_{1} X_{2} \sim S u b(1 /(N M))$. 
Proof: By definition of Rademacher RVs, we have:

$$
\begin{aligned}
X_{1} X_{2} & = \begin{cases}1 / \sqrt{M N} & \text { with } p_{1} \\
-1 / \sqrt{M N} & \text { with } p_{2}\end{cases} \\
p_{1} & =\operatorname{Pr}\left\{\left(X_{1}=1 / \sqrt{N}, X_{2}=1 / \sqrt{M}\right) \cup\left(X_{1}=-1 / \sqrt{N}, X_{2}=-1 / \sqrt{M}\right)\right\}=2 p^{2}=1 / 2 \\
p_{2} & =\operatorname{Pr}\left\{\left(X_{1}=-1 / \sqrt{N}, X_{2}=1 / \sqrt{M}\right) \cup\left(X_{1}=1 / \sqrt{N}, X_{2}=-1 / \sqrt{M}\right)\right\}=2 p^{2}=1 / 2
\end{aligned}
$$

From Definition 2 in Section II, we have:

$$
\begin{aligned}
\mathbb{E}\left[\exp \left(X_{1} X_{2} t\right)\right] & =\operatorname{Pr}\left\{X_{1} X_{2}=1 / \sqrt{M N}\right\} \exp (t / \sqrt{M N})+\operatorname{Pr}\left\{X_{1} X_{2}=-1 / \sqrt{M N}\right\} \exp (-t / \sqrt{M N}) \\
& =\cosh (t / \sqrt{M N})=\sum_{n \geq 0} \frac{(t / \sqrt{M N})^{2 n}}{(2 n) !} \stackrel{(a)}{\leq} \sum_{n \geq 0} \frac{\left((t / \sqrt{M N})^{2}\right)^{n}}{2^{n} n !}=\exp \left(\frac{t^{2}}{2 M N}\right)
\end{aligned}
$$

where (a) follows from the fact that $2^{n} n ! \geq(2 n)$ !, which can be proved by using the CauchySchwarz inequality. This concludes the proof.

\section{B. Lemma 2}

Let $X=\left[X_{1}, X_{2}, \ldots, X_{N}\right]^{T}$, where $X_{i}$ for $i=1,2, \ldots, N$ are i.i.d. subgaussian RVs such that $X_{i} \sim S u b\left(c^{2}\right)$. Then, $\sum_{i=1}^{N} X_{i} \sim S u b\left(N c^{2}\right)$.

Proof: Since $X_{i}$ are i.i.d. RVs, according to Definition 2 in Section II we have:

$$
\begin{aligned}
\mathbb{E}\left(\exp \left(t \sum_{i=1}^{N} X_{i}\right)\right) & =\mathbb{E}\left(\prod_{i=1}^{N} \exp \left(t X_{i}\right)\right) \\
& =\prod_{i=1}^{N} \mathbb{E}\left(\exp \left(t X_{i}\right)\right) \leq \prod_{i=1}^{N} \exp \left(c^{2}(t)^{2} / 2\right)=\exp \left(N c^{2} t^{2} / 2\right)
\end{aligned}
$$

Then, the proof follows from Definition 2.

\section{Proof of Proposition 1}

Initially, we need to identify the conditions under which both matrices $\mathbf{A}_{F W_{1}}$ and $\mathbf{A}_{R L N C_{1}}$ satisfy the RIP of the same order and with the same probability. 3.6 [40]], a matrix $\Phi$, of $\operatorname{rank} M<N$, will satisfy the RIP of order $K_{s}$ w.h.p., if the following conditions are satisfied: 1 ) the elements $\Phi_{i, j}$ of the matrix are i.i.d. subgaussian RVs,

i.e., $\left.\mathbb{E}\left[\exp \left(\Phi_{i, j} t\right)\right]=\exp \left(c^{2} \frac{t^{2}}{2}\right) 2\right)$ their distribution will yield a matrix that will be norm preserving, i.e., $\mathbb{E}\left[\|\mathbf{x}\|_{2}\right]=\|\mathbf{x}\|_{2}$ 3) The rank of $\Phi$, will satisfy the following inequality: $M \geq k_{1} K_{s} \log \left(\frac{N}{K_{s}}\right)$ where $k_{1}$ is an arbitrary value.

Recall, that the matrices $\mathbf{I}_{e_{11}}$ and $\mathbf{I}_{e_{12}}$ in eqs. (11), (13), (15) can be obtained from the identity matrix $\mathbf{I}_{M}$ after selecting the rows that correspond to the correctly received packets. 
Thus, the multiplication of matrices $\mathbf{I}_{e_{11}}$ and $\mathbf{I}_{e_{12}}$ with $\mathbf{A}_{s}$ and $\mathbf{P}_{r_{1}} \mathbf{I}_{e_{11}} \mathbf{A}_{s}$ in the $\mathrm{FW}$ case and with $\mathbf{A}_{s}$ and $\mathbf{A}_{r_{1}} \mathbf{I}_{e_{11}} \mathbf{A}_{s}$ in the RLNC case results in the elimination of a random subset of their rows. Each row is deleted Let $p_{e_{1}}, p_{e_{2}}$ be the packet error probabilities in the links $S \rightarrow r_{1}$ and $r_{1} \rightarrow B N C$. Then the number of rows deleted in each link would be on average equal to $p_{e_{1}} M$ and $p_{e_{2}} M$ respectively.

In the FW case, the matrix $\mathbf{P}_{r_{1}}$ that is applied at the relay simply repeats in a round robin fashion the rows of the matrix $\mathbf{I}_{e_{11}} \mathbf{A}_{s}$, which consists of Rademacher elements. This means that $a=\bmod \left(M,\left(1-p_{e_{1}}\right) M\right)$ packets are transmitted $\left\lfloor M /\left(1-p_{e_{1}}\right) M\right\rfloor+1$ times and $\left(1-p_{e_{1}}\right) M-a$ packets are transmitted $\left\lfloor M /\left(1-p_{e_{1}}\right) M\right\rfloor$ times. The average number of packets received at the $\mathrm{BNC}$ is equal to $\left[a\left(1-p_{e}^{b+1}\right)+(K-a)\left(1-p_{e}^{b}\right)\right]$. As a result, the rank of matrix $\mathbf{A}_{F W_{1}}$ would be on average equal to $M_{F W}=\left[a\left(1-p_{e}^{b+1}\right)+(K-a)\left(1-p_{e}^{b}\right)\right]$, and its elements will be Rademacher elements which are i.i.d. subgaussian RVs, i.e., $\mathbf{A}_{F W_{1} i, j} \sim$ Sub(1/M). According to [40, Corollary 3.1], this matrix also be norm preserving.

In the RLNC case, the end to end encoding matrix is $\mathbf{A}_{R L N C}=\mathbf{A}_{1} \mathbf{A}_{2}$ where $\mathbf{A}_{1}=\mathbf{I}_{e_{12}} \mathbf{A}_{r}$ and $\mathbf{A}_{2}=\mathbf{I}_{e_{11}} \mathbf{A}_{s}$ are Rademacher matrices of average sizes $\left(1-p_{e_{2}}\right) M \times\left(1-p_{e_{1}}\right) M$ and $\left(1-p_{e_{1}}\right) M \times N$. With the aid of Lemmas 1, each element of $\mathbf{A}_{R L N C}$ can be written as:

$$
\mathbf{A}_{R L N C_{i, j}}=\sum_{k=1}^{\left(1-p_{e_{1}}\right) M} \mathbf{A}_{1_{i, k}} \mathbf{A}_{2_{k, j}}=\sum_{k=1}^{\left(1-p_{e_{1}}\right) M} X_{k}
$$

where $X_{k} \sim S u b\left(1 / M^{2}\right)$, since $\mathbf{A}_{1_{i, j}} \sim S u b(1 / M), \mathbf{A}_{2_{i, j}} \sim S u b(1 / M) \forall i, j$. Based on Lemma 2, that $\mathbf{A}_{R L N C_{i, j}} \sim \operatorname{Sub}\left(\left(1-p_{e_{1}}\right) / M\right)$. account that the elements of matrices $\mathbf{A}_{1}, \mathbf{A}_{2}$ are i.i.d. RVs, it can be easily verified that the covariance matrix $\frac{1}{\left(1-p_{e_{1}}\right)\left(1-p_{e_{2}}\right)} \mathbb{E}\left[\mathbf{A}_{2}^{T} \mathbf{A}_{1}^{T} \mathbf{A}_{1} \mathbf{A}_{2}\right]$ tends to the identity since the diagonal elements have mean 1 and the off diagonal have variance $O(1 / M)$. In other words the elements of $\mathbf{A}_{R L N C}$ are i.i.d. and the matrix $\frac{1}{\sqrt{\left(1-p_{e_{1}}\right)\left(1-p_{e_{2}}\right)}} \mathbf{A}_{R L N C_{i, j}}$ is norm preserving. Finally, it can be exsily verified that the rank of $\mathbf{A}_{R L N C}$ on average equal to $M_{R L N C}=\min \left(\left(1-p_{e_{1}}\right) M,\left(1-p_{e_{2}}\right) M\right)$.

Since, both $\mathbf{A}_{R L N C}$ and $\mathbf{A}_{F W}$ satisfy conditions 1,2, it remains to identify when condition 3 is satisfied. To ensure that the encoding matrix, in both cases (RLNC \& FW) will be equal

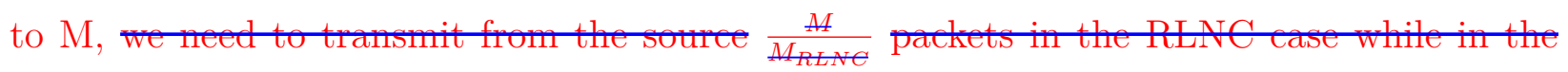

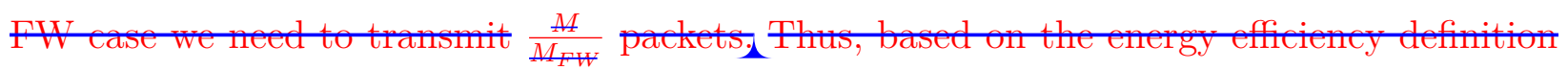
in energen efficiency gains of RLNC as compared to the FW case, 
can be calculated as the ratio $\frac{M_{R L N C}}{M_{F W}}$. This concludes the proof.

\section{Proof of Proposition 2}

In phases $I$, the matrix $\mathbf{I}_{e_{i 1}}$ is formed by randomly deleting rows of $\mathbf{A}_{s}$ with probability $p_{e_{1}}$. As a result, a row does not exist in any of the matrices $\mathbf{I}_{e_{i 1}} \mathbf{A}_{s}$ for $\forall i=1, \ldots, n$, if it has been deleted by all matrices $\mathbf{I}_{e_{i 1}}$. This event occurs with probability $p_{e_{1}}^{n}$, since there is no correlation (either in space or in time) between the error probability in different links. Thus, in the FW case, the multirelay system can be treated as a single relay one with error probabilities $p_{e_{1}}^{n}$ and $p_{e_{2}}^{n}$ respectively. In the RLNC case, the received packets $\mathbf{y}_{R L N C}$ may be written as follows:

$$
\mathbf{y}_{R L N C}=\underbrace{\left[\begin{array}{cccc}
\mathbf{I}_{e_{12}} \mathbf{A}_{r_{1}} & \mathbf{0}_{d} & \ldots & \mathbf{0}_{d} \\
\ldots & \ddots & \ddots & \ldots \\
\mathbf{0}_{d} & \ldots & \ldots & \mathbf{I}_{e_{n 2}} \mathbf{A}_{r_{n}}
\end{array}\right]}_{\mathbf{A}_{\mathbf{2}}} \underbrace{\left[\begin{array}{c}
\mathbf{I}_{e_{11}} \mathbf{A}_{s} \\
\ldots \\
\mathbf{I}_{e_{n 1}} \mathbf{A}_{s}
\end{array}\right]}_{\mathbf{A}_{\mathbf{1}}} \mathbf{x}+\mathbf{z}_{R L N C},
$$

It can be easily verified that $\mathbf{A}_{R L N C}$ consist of i.i.d. subgaussian RVs and the rank of $\mathbf{A}_{R L N C}$ be on average equal to $M_{R L N C}=\min \left(\left(1-p_{e_{1}}^{n}\right) M, n\left(1-p_{e_{2}}\right) M\right)$, since the rank of matrices $\mathbf{A}_{1}$ and $\mathbf{A}_{2}$ are on average equal to $\left(1-p_{e_{1}}^{n}\right) M$ and $n\left(1-p_{e_{2}}\right) M$ respectively. To ensure that the encoding matrix, in both cases (RLNC \& FW) equal to M,

$\frac{M}{M_{R L N C}}$ and $\frac{M}{M_{F W}}$, respectively. Thus the energy efficiency gains of RLNC as compared to the FW ease, are equal to $\frac{M_{R L N C}}{M_{F W}}$. This concludes the proof.

\section{REFERENCES}

[1] H. Cao, V. Leung, C. Chow, and H. Chan, "Enabling technologies for wireless body area networks: A survey and outlook," IEEE Commun. Mag., vol. 47, no. 12, pp. 84-93, 2009.

[2] Y. Chen et al., "Cooperative communications in ultra-wideband wireless body area networks: Channel modeling and system diversity analysis," IEEE J. Sel. Areas Commun, vol. 27, no. 1, pp. 5-16, January 2009.

[3] A. Dixon et al., "Compressed sensing system considerations for ecg and emg wireless biosensors," IEEE Trans. Biomed. Circuits Syst., vol. 6, no. 2, pp. 156-166, 2012.

[4] S. Movassaghi et al., "Wireless body area networks: A survey," IEEE Commun. Surveys Tuts., vol. PP, no. 99, pp. 1-29, 2014.

[5] J. Dong and D. Smith, "Opportunistic relaying in wireless body area networks: Coexistence performance," in IEEE Int. Conf. on Commun. (ICC), 2013, June 2013, pp. 5613-5618.

[6] L. Srnmo and P. Laguna, Bioelectrical Signal Processing in Cardiac and Neurological Applications. Elsevier/Academic press: New York, 2005. 
[7] S. M. S. Jalaleddine, C. Hutchens, R. Strattan, and W. Coberly, "Ecg data compression techniques-a unified approach," IEEE Transactions on Biomedical Engineering, vol. 37, no. 4, pp. 329-343, 1990.

[8] M. Abo-Zahhad, A. F. Al-Ajlouni, S. M. Ahmed, and R. Schilling, "A new algorithm for the compression of \{ECG\} signals based on mother wavelet parameterization and best-threshold levels selection," Digital Signal Processing, vol. 23, no. 3, pp. 1002 - 1011, 2013.

[9] A. Boulis, D. Smith, D. Miniutti, L. Libman, and Y. Tselishchev, "Challenges in body area networks for healthcare: the mac," IEEE Communications Magazine, vol. 50, no. 5, pp. 100-106, May 2012.

[10] W. Chen, M. Rodrigues, and I. Wassell, "A frechet mean approach for compressive sensing date acquisition and reconstruction in wireless sensor networks," IEEE Transactions on Wireless Communications, vol. 11, no. 10, pp. 3598-3606, October 2012.

[11] J. Cheng, Q. Ye, H. Jiang, D. Wang, and C. Wang, "Stcdg: An efficient data gathering algorithm based on matrix completion for wireless sensor networks," IEEE Transactions on Wireless Communications, vol. 12, no. 2, pp. 850-861, February 2013.

[12] G. Quer, R. Masiero, G. Pillonetto, M. Rossi, and M. Zorzi, "Sensing, compression, and recovery for wsns: Sparse signal modeling and monitoring framework," IEEE Transactions on Wireless Communications, vol. 11, no. 10, pp. 3447-3461, October 2012.

[13] H. Mamaghanian et al., "Compressed sensing for real-time energy-efficient ecg compression on wireless body sensor nodes," IEEE Trans. Biomed. Eng, vol. 58, no. 9, pp. 2456-2466, 2011.

[14] Z. Zhang et al., "Compressed sensing for energy-efficient wireless telemonitoring of noninvasive fetal ecg via block sparse bayesian learning," IEEE Trans. Biomed. Eng, vol. 60, no. 2, pp. 300-309, 2013.

[15] X. Shi, M. Medard, and D. Lucani, "Whether and where to code in the wireless packet erasure relay channel," IEEE Journal on Selected Areas in Communications, vol. 31, no. 8, pp. 1379-1389, Aug 2013.

[16] C. Luo, J. Sun, and F. Wu, "Compressive network coding for approximate sensor data gathering," in IEEE Global Telecommunications Conference (GLOBECOM 2011), Dec 2011, pp. 1-6.

[17] N. Nguyen, D. Jones, and S. Krishnamurthy, "Netcompress: Coupling network coding and compressed sensing for efficient data communication in wireless sensor networks," in IEEE Workshop on Signal Processing Systems (SIPS), Oct 2010, pp. 356-361.

[18] X. Yang et al., "Energy-efficient distributed data storage for wireless sensor networks based on compressed sensing and network coding," IEEE Transactions on Wireless Communications, vol. 12, no. 10, pp. 5087-5099, October 2013.

[19] D. Donoho, "Compressed sensing," IEEE Trans. Inf. Theory, vol. 52, no. 4, pp. 1289-1306, 2006.

[20] E. Candes and T. Tao, "Near-optimal signal recovery from random projections: Universal encoding strategies?" IEEE Trans. Inf. Theory, vol. 52, no. 12, pp. 5406-5425, 2006.

[21] E. Candes and M. Wakin, "An introduction to compressive sampling," IEEE Signal Process. Mag., vol. 25, no. 2, pp. 21-30, 2008.

[22] S. Mendelson, A. Pajor, and N. Tomczak-Jaegermann, "Uniform uncertainty principle for bernoulli and subgaussian ensembles," Constructive Approximation, vol. 28, no. 3, pp. 277-289, 2008.

[23] J. Friedman, T. Hastie, H. Hfling, and R. Tibshirani, "Pathwise coordinate optimization," Ann. Appl. Stat., Tech. Rep., 2007. 
[24] R. Baraniuk, V. Cevher, M. Duarte, and C. Hegde, "Model-based compressive sensing," Information Theory, IEEE Transactions on, vol. 56, no. 4, pp. 1982-2001, April 2010.

[25] R. Northrop, Analysis and Application of Analog Electronic Circuits to Biomedical Instrumentation, ser. Biomedical Engineering. CRC Press, 2004. [Online]. Available: http://books.google.es/books?id=CSOva1nQsUEC

[26] S. Chachulski, M. Jennings, S. Katti, and D. Katabi, "Trading structure for randomness in wireless opportunistic routing," SIGCOMM Comput. Commun. Rev., vol. 37, no. 4, pp. 169-180, Aug. 2007. [Online]. Available: http://doi.acm.org/10.1145/1282427.1282400

[27] H. Seferoglu, A. Markopoulou, and K. Ramakrishnan, "I2nc: Intra- and inter-session network coding for unicast flows in wireless networks," in INFOCOM, 2011 Proceedings IEEE, April 2011, pp. 1035-1043.

[28] Y. Eldar, P. Kuppinger, and H. Bolcskei, "Block-sparse signals: Uncertainty relations and efficient recovery," IEEE Trans. Signal Process, vol. 58, no. 6, pp. 3042-3054, 2010.

[29] M. Stojnic, F. Parvaresh, and B. Hassibi, "On the reconstruction of block-sparse signals with an optimal number of measurements," IEEE Trans. Signal Process, vol. 57, no. 8, pp. 3075-3085, 2009.

[30] R. Rubinstein, A. Bruckstein, and M. Elad, "Dictionaries for sparse representation modeling," Proceedings of the IEEE, vol. 98, no. 6, pp. 1045-1057, June 2010.

[31] M. Nielsen, E. Kamavuako, M. Andersen, M.-F. Lucas, and D. Farina, "Optimal wavelets for biomedical signal compression," Medical and Biological Engineering and Computing, vol. 44, no. 7, pp. 561-568, 2006. [Online]. Available: http://dx.doi.org/10.1007/s11517-006-0062-0

[32] S. Mukhopadhyay and P. Sircar, "Parametric modelling of ecg signal," Medical and Biological Engineering and Computing, vol. 34, no. 2, pp. 171-174, 1996. [Online]. Available: http://dx.doi.org/10.1007/BF02520024

[33] G. AL, A. LAN, G. L, H. JM, I. PCh, M. RG, M. JE, M. GB, P. C-K, and S. HE, "Physiobank, physiotoolkit, and physionet: Components of a new research resource for complex physiologic signals," Circulation, vol. 100, no. 23, pp. e215-e220, Jun 2013.

[34] E. Cands, M. B. Wakin, and S. P. Boyd, "Enhancing sparsity by reweighted $\ell_{1}$ minimization," Journal of Fourier Analysis and Applications, vol. 14, no. 5-6, pp. 877-905, 2008.

[35] D. Wipf and S. Nagarajan, "Iterative reweighted $\ell_{1}$ and $\ell_{2}$ methods for finding sparse solutions," IEEE Journal of Selected Topics in Signal Processing, vol. 4, no. 2, pp. 317-329, 2010.

[36] R. Chartrand and W. Yin, "Iteratively reweighted algorithms for compressive sensing," in Acoustics, Speech and Signal Processing, 2008. ICASSP 2008. IEEE International Conference on, March 2008, pp. 3869-3872.

[37] LAN/MAN Standards Committee of the IEEE Computer Society, "IEEE Standard for Local and metropolitan area networks - Part 15.6: Wireless Body Area Networks," IEEE Std 802.15.6-2012, Feb. 2012.

[38] Y. Zigel, A. Cohen, and A. Katz, "The weighted diagnostic distortion (wdd) measure for ecg signal compression," IEEE Trans. Biomed. Eng, vol. 47, no. 11, pp. 1422-1430, 2000.

[39] R. G. Andrzejak, K. Lehnertz, F. Mormann, C. Rieke, P. David, and C. E. Elger, "Indications of nonlinear deterministic and finite-dimensional structures in time series of brain electrical activity: dependence on recording region and brain state." Physical review. E, Statistical, nonlinear, and soft matter physics, vol. 64, no. 6 Pt 1, Dec. 2001.

[40] M. D. R. Baraniuk, M. Davenport and C. Hegde, An Introduction to Compressive Sensing, ser. An Introduction to Compressive Sensing. Addison-Wesley, 2011. 